\title{
Cucurbalsaminones A-C, Rearranged Triterpenoids with a 5/6/3/6/5-Fused Pentacyclic Carbon Skeleton from Momordica balsamina as Multidrug Resistance Reversers
}

Andreia Mónico ${ }^{\dagger}$ Cátia Ramalhete,${ }^{\dagger}{ }^{\ddagger}$ Vânia André,${ }^{\S}$ Gabriella Spengler, ${ }^{\perp}$ Silva Mulhovo, ${ }^{\dagger}$ M. Teresa Duarte, ${ }^{\S}$ and Maria-José U. Ferreira ${ }^{*} \uparrow$

$\dagger$ Research Institute for Medicines (iMed.ULisboa), Faculty of Pharmacy, Universidade de Lisboa, Av. Prof. Gama Pinto, 1649-003 Lisbon, Portugal

‡ATLÂNTICA - Escola Universitária de Ciências Empresariais, Saúde, Tecnologias e Engenharia, Fábrica da Pólvora de Barcarena, 2730-036 Barcarena, Oeiras, Portugal

${ }^{\S}$ Centro de Química Estrutural, Instituto Superior Técnico, Universidade de Lisboa, Av. Rovisco Pais, 1049-001 Lisbon, Portugal

${ }^{\perp}$ Department of Medical Microbiology and Immunobiology, Faculty of Medicine, University of Szeged, Dóm tér 10, H-6720 Szeged, Hungary

${ }^{1}$ Centro de Estudos Moçambicanos e de Etnociências, Faculdade de Ciências e Matemática, Universidade Pedagógica, 21402161 Maputo, Mozambique 


\section{X-ray Crystallography}

Table S1. Crystal data and structure refinement details for 1 (CCDC 1878164).

\begin{tabular}{lc}
\hline Formula & $\mathrm{C}_{27} \mathrm{H}_{40} \mathrm{O}_{3}$ \\
Fw & 412.59 \\
Crystal form, color & Block, colourless \\
Crystal size (mm) & $0.20 \times 0.10 \times 0.04$ \\
cryst. syst. & Orthorhombic \\
space group & $P 2_{1} 2_{1} 2_{1}$ \\
$a, \AA$ & $6.1959(4)$ \\
$b, \AA$ & $14.0177(8)$ \\
$c, \AA$ & $26.2372(16)$ \\
$Z$ & 4 \\
$V, \AA{ }^{3}$ & $2278.8(2)$ \\
$T, \mathrm{~K}$ & $150(2)$ \\
$D_{\mathrm{c}}, \mathrm{g} \mathrm{cm}{ }^{-3}$ & 1.203 \\
$\mu($ Mo K $\alpha), \mathrm{mm}^{-1}$ & 0.076 \\
$\theta$ range $\left({ }^{\circ}\right)$ & $2.745-26.436$ \\
refl. Collected & 26968 \\
independent refl. & 4682 \\
$R_{\text {int }}$ & 0.0813 \\
$R_{1}{ }^{a}, w R_{2}{ }^{b}[I \geq 2 \sigma(I)]$ & $0.0496,0.1140$ \\
$\mathrm{GOF}$ on $F^{2}$ & 1.013 \\
\hline${ }^{a} R_{1}=\left.\Sigma|| F_{\mathrm{o}}|-| F_{\mathrm{c}}|/ \Sigma| F_{\mathrm{o}}\right|^{b}{ }^{b} w R_{2}=\left[\Sigma\left[w\left(F_{\mathrm{o}}{ }^{2}-F_{\mathrm{c}}{ }^{2}\right)^{2}\right] / \Sigma\left[w\left(F_{\mathrm{o}}{ }^{2}\right)^{2}\right]\right]^{1 / 2}$
\end{tabular}


Table S2. Bond lengths $[\AA]$ and angles $\left[^{\circ}\right]$ for compound 1.

\begin{tabular}{|c|c|c|c|c|c|}
\hline $\mathrm{O}(2)-\mathrm{C}(7)$ & $1.214(4)$ & $\mathrm{C}(12)-\mathrm{C}(13)-\mathrm{C}(18)$ & $110.1(2)$ & $\mathrm{C}(3)-\mathrm{C}(5)-\mathrm{C}(4)$ & $119.9(3)$ \\
\hline $\mathrm{O}(3)-\mathrm{C}(23)$ & $1.194(4)$ & $\mathrm{C}(12)-\mathrm{C}(13)-\mathrm{C}(17)$ & $117.5(2)$ & $C(6)-C(5)-C(4)$ & $59.2(2)$ \\
\hline$C(13)-C(12)$ & $1.524(4)$ & $\mathrm{C}(18)-\mathrm{C}(13)-\mathrm{C}(17)$ & $108.1(2)$ & $C(10)-C(5)-C(4)$ & $123.3(3)$ \\
\hline $\mathrm{C}(13)-\mathrm{C}(18)$ & $1.544(4)$ & $\mathrm{C}(12)-\mathrm{C}(13)-\mathrm{C}(14)$ & $107.1(2)$ & $\mathrm{C}(19)-\mathrm{C}(9)-\mathrm{C}(11)$ & $106.8(2)$ \\
\hline$C(13)-C(17)$ & $1.558(4)$ & $\mathrm{C}(18)-\mathrm{C}(13)-\mathrm{C}(14)$ & $112.2(2)$ & $C(19)-C(9)-C(10)$ & $108.6(2)$ \\
\hline$C(13)-C(14)$ & $1.571(4)$ & $\mathrm{C}(17)-\mathrm{C}(13)-\mathrm{C}(14)$ & $101.6(2)$ & $\mathrm{C}(11)-\mathrm{C}(9)-\mathrm{C}(10)$ & 109.7(3) \\
\hline $\mathrm{C}(8)-\mathrm{C}(7)$ & $1.532(4)$ & $\mathrm{C}(7)-\mathrm{C}(8)-\mathrm{C}(14)$ & $111.6(2)$ & $\mathrm{C}(19)-\mathrm{C}(9)-\mathrm{C}(8)$ & $106.9(3)$ \\
\hline $\mathrm{C}(8)-\mathrm{C}(14)$ & $1.560(4)$ & $\mathrm{C}(7)-\mathrm{C}(8)-\mathrm{C}(9)$ & $114.5(2)$ & $\mathrm{C}(11)-\mathrm{C}(9)-\mathrm{C}(8)$ & $113.2(2)$ \\
\hline $\mathrm{C}(8)-\mathrm{C}(9)$ & $1.584(4)$ & $\mathrm{C}(14)-\mathrm{C}(8)-\mathrm{C}(9)$ & $117.1(2)$ & $\mathrm{C}(10)-\mathrm{C}(9)-\mathrm{C}(8)$ & $111.5(2)$ \\
\hline$C(17)-C(20)$ & $1.534(4)$ & $\mathrm{C}(20)-\mathrm{C}(17)-\mathrm{C}(13)$ & $119.5(2)$ & $\mathrm{O}(3)-\mathrm{C}(23)-\mathrm{C}(24)$ & $121.5(3)$ \\
\hline$C(17)-C(16)$ & $1.571(4)$ & $\mathrm{C}(20)-\mathrm{C}(17)-\mathrm{C}(16)$ & $112.0(2)$ & $\mathrm{O}(3)-\mathrm{C}(23)-\mathrm{C}(22)$ & $121.8(3)$ \\
\hline$C(20)-C(21)$ & $1.521(4)$ & $\mathrm{C}(13)-\mathrm{C}(17)-\mathrm{C}(16)$ & $102.7(2)$ & $C(24)-C(23)-C(22)$ & $116.6(3)$ \\
\hline$C(20)-C(22)$ & $1.535(4)$ & $\mathrm{C}(21)-\mathrm{C}(20)-\mathrm{C}(17)$ & $114.3(2)$ & $\mathrm{C}(15)-\mathrm{C}(16)-\mathrm{C}(17)$ & $107.5(2)$ \\
\hline$C(7)-C(6)$ & $1.493(4)$ & $\mathrm{C}(21)-\mathrm{C}(20)-\mathrm{C}(22)$ & $108.5(3)$ & $C(2)-C(1)-C(10)$ & $107.6(3)$ \\
\hline$C(10)-C(5)$ & $1.522(4)$ & $\mathrm{C}(17)-\mathrm{C}(20)-\mathrm{C}(22)$ & $108.3(2)$ & $C(14)-C(15)-C(16)$ & $103.9(2)$ \\
\hline $\mathrm{C}(10)-\mathrm{C}(1)$ & $1.558(4)$ & $\mathrm{O}(2)-\mathrm{C}(7)-\mathrm{C}(6)$ & $118.2(3)$ & $\mathrm{C}(12)-\mathrm{C}(11)-\mathrm{C}(9)$ & $118.9(2)$ \\
\hline $\mathrm{C}(10)-\mathrm{C}(9)$ & $1.565(4)$ & $\mathrm{O}(2)-\mathrm{C}(7)-\mathrm{C}(8)$ & $120.8(3)$ & $\mathrm{C}(23)-\mathrm{C}(22)-\mathrm{C}(20)$ & $114.6(3)$ \\
\hline $\mathrm{C}(12)-\mathrm{C}(11)$ & $1.528(4)$ & $\mathrm{C}(6)-\mathrm{C}(7)-\mathrm{C}(8)$ & $120.8(3)$ & $\mathrm{C}(3)-\mathrm{C}(2)-\mathrm{C}(1)$ & 106.1(3) \\
\hline $\mathrm{C}(6)-\mathrm{C}(5)$ & $1.506(4)$ & $\mathrm{C}(5)-\mathrm{C}(10)-\mathrm{C}(1)$ & $102.9(2)$ & $\mathrm{O}(1)-\mathrm{C}(3)-\mathrm{C}(5)$ & $126.1(3)$ \\
\hline $\mathrm{C}(6)-\mathrm{C}(4)$ & $1.508(4)$ & $\mathrm{C}(5)-\mathrm{C}(10)-\mathrm{C}(9)$ & $110.9(3)$ & $\mathrm{O}(1)-\mathrm{C}(3)-\mathrm{C}(2)$ & 125.1(3) \\
\hline$C(14)-C(30)$ & $1.536(4)$ & $\mathrm{C}(1)-\mathrm{C}(10)-\mathrm{C}(9)$ & $115.3(2)$ & $C(5)-C(3)-C(2)$ & $108.8(3)$ \\
\hline$C(14)-C(15)$ & $1.537(4)$ & $\mathrm{C}(13)-\mathrm{C}(12)-\mathrm{C}(11)$ & $110.9(3)$ & $C(29)-C(4)-C(6)$ & $122.8(3)$ \\
\hline $\mathrm{C}(5)-\mathrm{C}(3)$ & $1.486(4)$ & $C(7)-C(6)-C(5)$ & $118.4(3)$ & $\mathrm{C}(29)-\mathrm{C}(4)-\mathrm{C}(28)$ & $111.8(3)$ \\
\hline $\mathrm{C}(5)-\mathrm{C}(4)$ & $1.548(4)$ & $C(7)-C(6)-C(4)$ & $124.3(3)$ & $\mathrm{C}(6)-\mathrm{C}(4)-\mathrm{C}(28)$ & 115.1(3) \\
\hline $\mathrm{C}(9)-\mathrm{C}(19)$ & $1.543(4)$ & $C(5)-C(6)-C(4)$ & $61.8(2)$ & $C(29)-C(4)-C(5)$ & $120.2(3)$ \\
\hline $\mathrm{C}(9)-\mathrm{C}(11)$ & $1.557(4)$ & $\mathrm{C}(30)-\mathrm{C}(14)-\mathrm{C}(15)$ & 106.7(2) & $C(6)-C(4)-C(5)$ & $59.0(2)$ \\
\hline$C(23)-C(24)$ & $1.494(5)$ & $\mathrm{C}(30)-\mathrm{C}(14)-\mathrm{C}(8)$ & $110.2(2)$ & $\mathrm{C}(28)-\mathrm{C}(4)-\mathrm{C}(5)$ & 118.7(3) \\
\hline$C(23)-C(22)$ & $1.504(5)$ & $\mathrm{C}(15)-\mathrm{C}(14)-\mathrm{C}(8)$ & $116.0(2)$ & & \\
\hline$C(16)-C(15)$ & $1.543(5)$ & $\mathrm{C}(30)-\mathrm{C}(14)-\mathrm{C}(13)$ & $112.8(2)$ & & \\
\hline $\mathrm{C}(1)-\mathrm{C}(2)$ & $1.537(5)$ & $\mathrm{C}(15)-\mathrm{C}(14)-\mathrm{C}(13)$ & $101.2(2)$ & & \\
\hline $\mathrm{O}(1)-\mathrm{C}(3)$ & $1.211(4)$ & $\mathrm{C}(8)-\mathrm{C}(14)-\mathrm{C}(13)$ & $109.9(2)$ & & \\
\hline $\mathrm{C}(2)-\mathrm{C}(3)$ & $1.501(5)$ & $\mathrm{C}(3)-\mathrm{C}(5)-\mathrm{C}(6)$ & $120.2(3)$ & & \\
\hline $\mathrm{C}(28)-\mathrm{C}(4)$ & $1.519(4)$ & $C(3)-C(5)-C(10)$ & $108.9(3)$ & & \\
\hline $\mathrm{C}(29)-\mathrm{C}(4)$ & $1.507(5)$ & $C(6)-C(5)-C(10)$ & $117.9(2)$ & & \\
\hline
\end{tabular}


Table S3. Antiproliferative activity of compounds $\mathbf{1}$ - $\mathbf{3}$ on $A B C B 1$-transfected mouse T-lymphoma (L5178Y) cells.

\begin{tabular}{lcc}
\hline compound & $\mathbf{P A R}^{a} \mathbf{I D}_{\mathbf{5 0}}(\boldsymbol{\mu M})$ & $\mathbf{M D R}^{a} \mathbf{I D}_{\mathbf{5 0}}(\boldsymbol{\mu M})$ \\
\hline cucurbalsaminone A (1) & $72.2 \pm 1.3$ & $68.3 \pm 2.1$ \\
cucurbalsaminone B (2) & $>100$ & $>100$ \\
cucurbalsaminone C (3) & $20.7 \pm 2.7$ & $37.6 \pm 3.9$ \\
\hline
\end{tabular}

${ }^{a}$ Values represent the mean \pm SD of three independent experiments 
HRMS, IR and NMR spectra of compounds 1-3

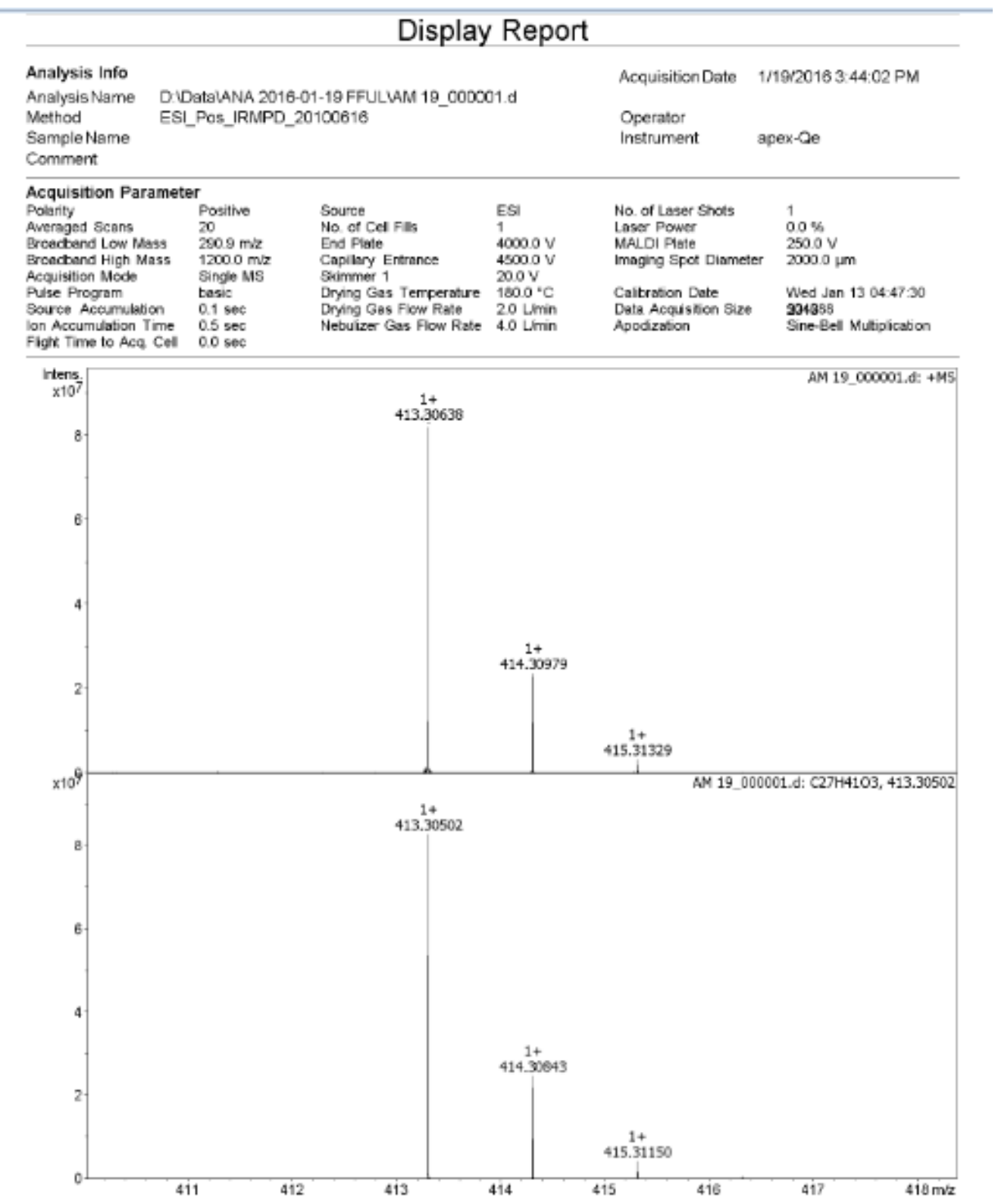

Figure S1. HRMS of compound 1. 
ФSHIMADZU

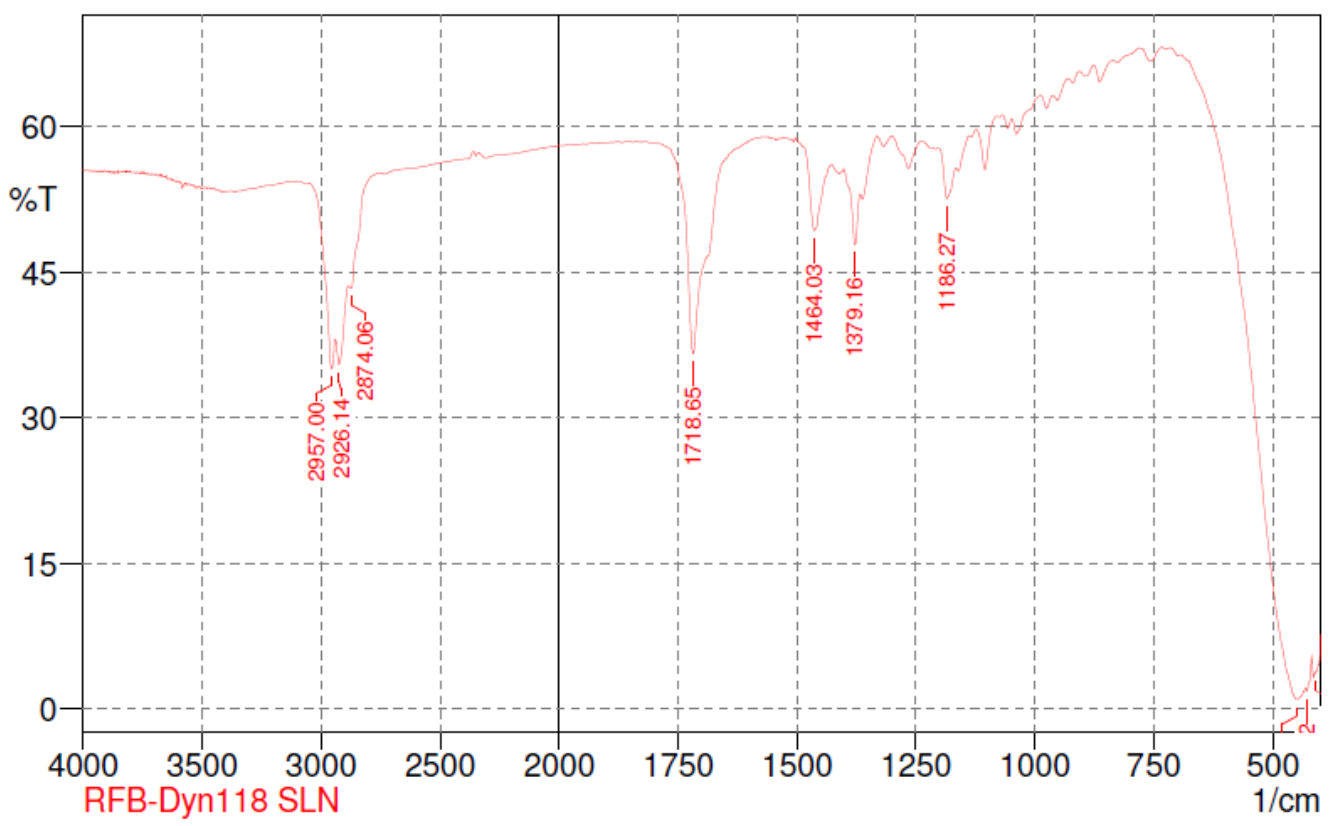

Figure S2. IR of compound 1.

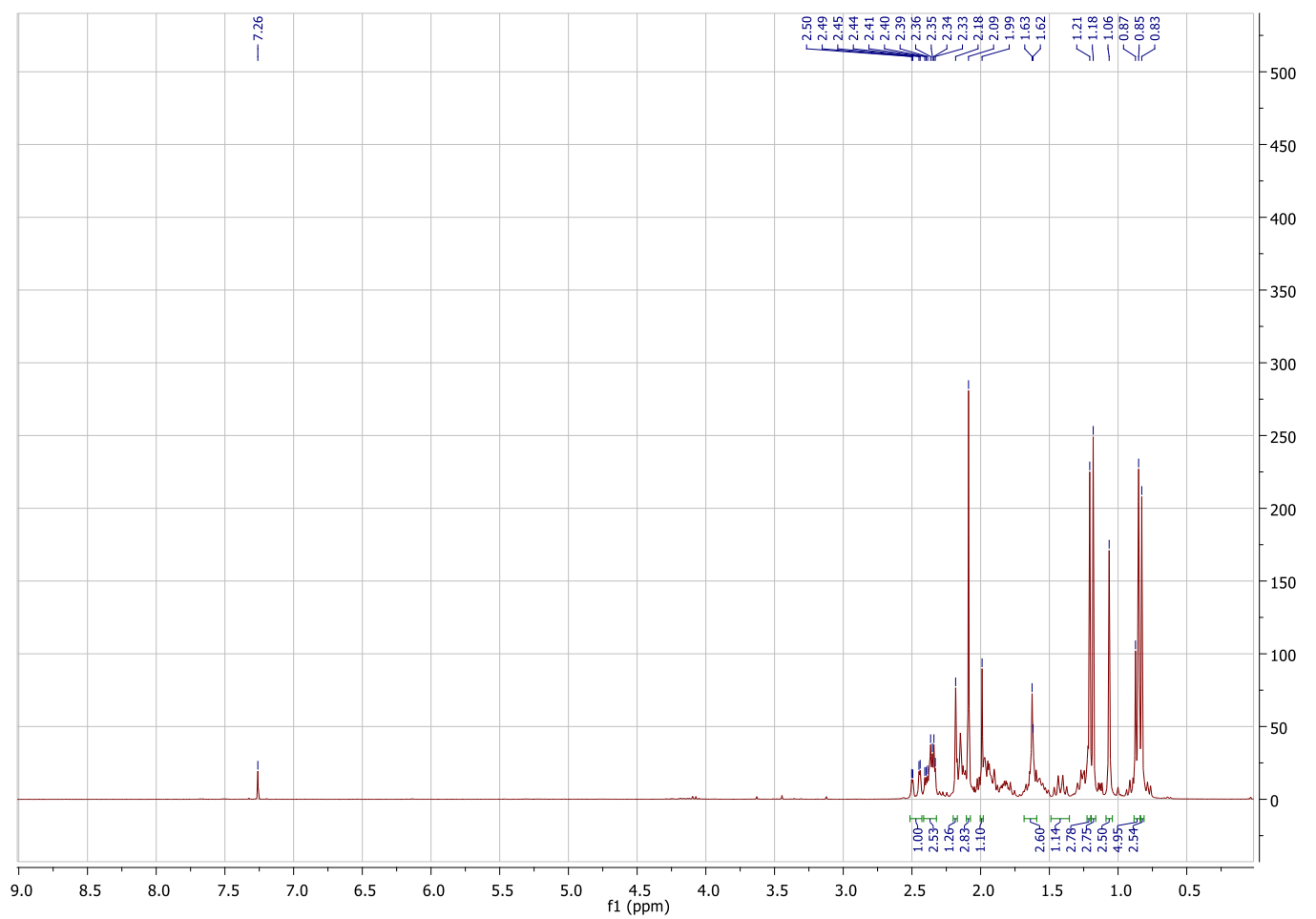

Figure S3. ${ }^{1} \mathrm{H}-\mathrm{NMR}$ spectrum of compound 1, in $\mathrm{CDCl}_{3}(300 \mathrm{MHz})$. 


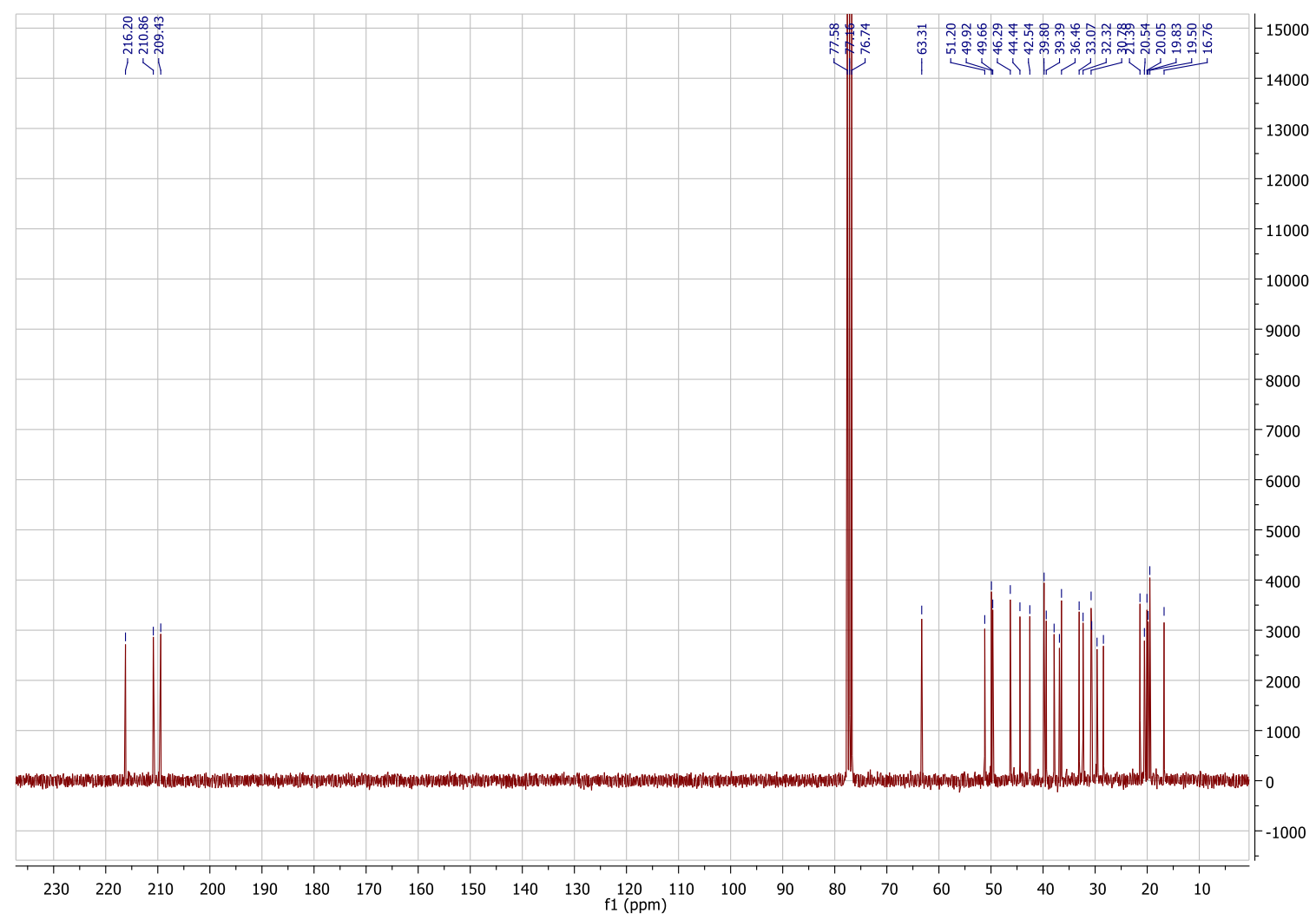

Figure S4. ${ }^{13} \mathrm{C}-\mathrm{NMR}$ spectrum of compound $\mathbf{1}$, in $\mathrm{CDCl}_{3}(75 \mathrm{MHz})$.

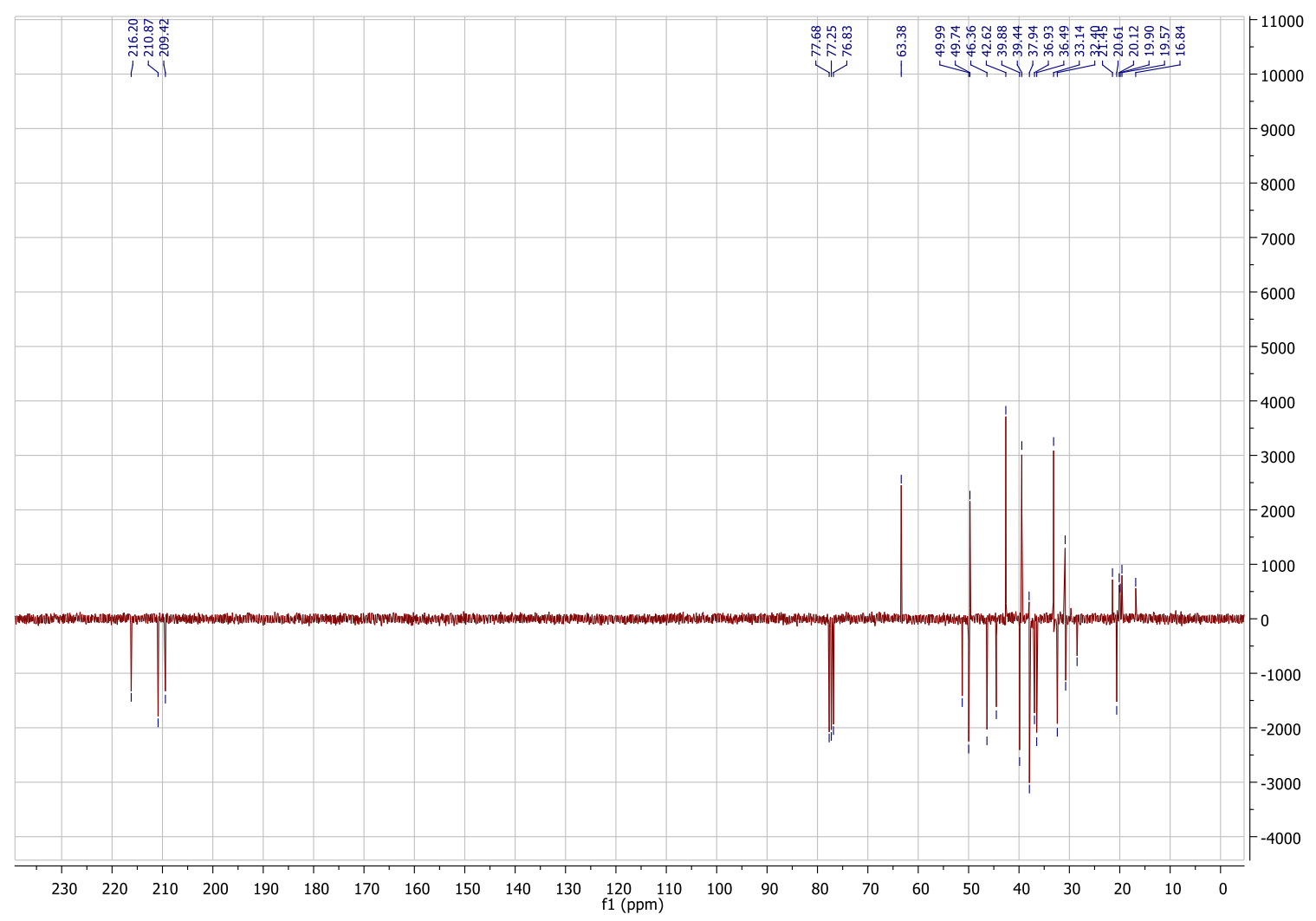

Figure S5. DEPT spectrum of compound 1 in $\mathrm{CDCl}_{3}(75 \mathrm{MHz})$. 


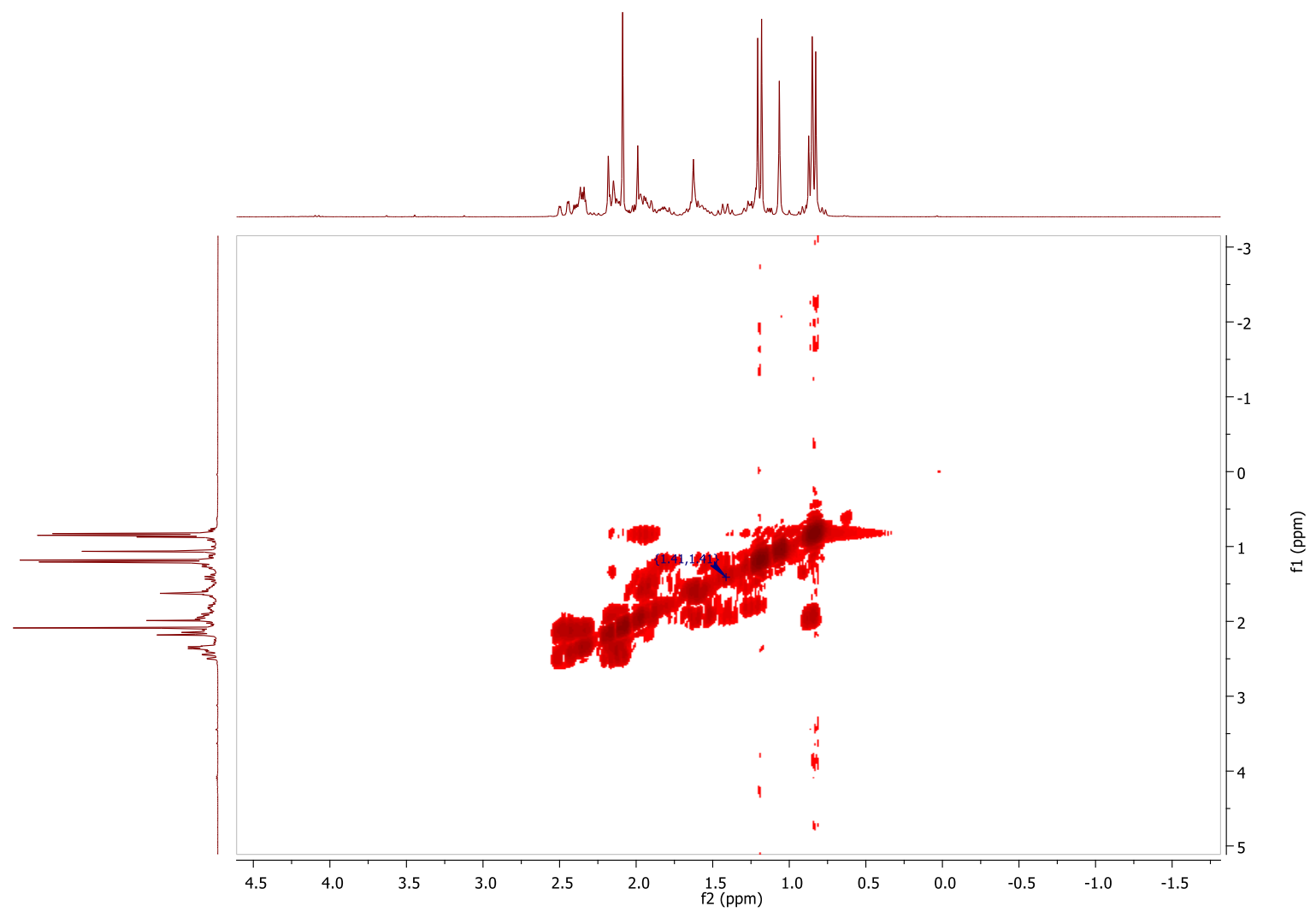

Figure S6. COSY spectrum of compound $\mathbf{1}$ in $\mathrm{CDCl}_{3}$.

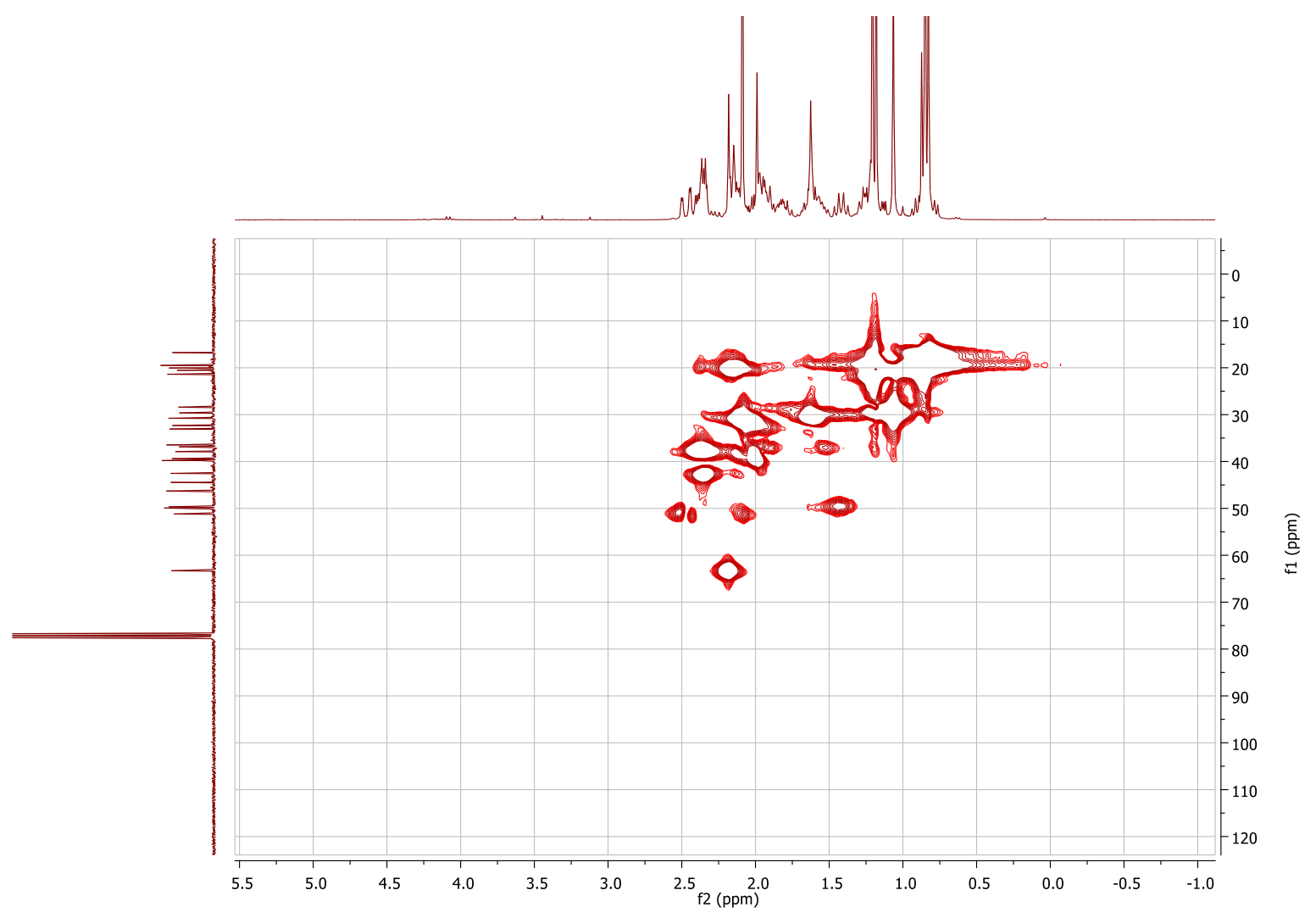

Figure S7. HMQC spectrum of compound 1 in $\mathrm{CDCl}_{3}$. 


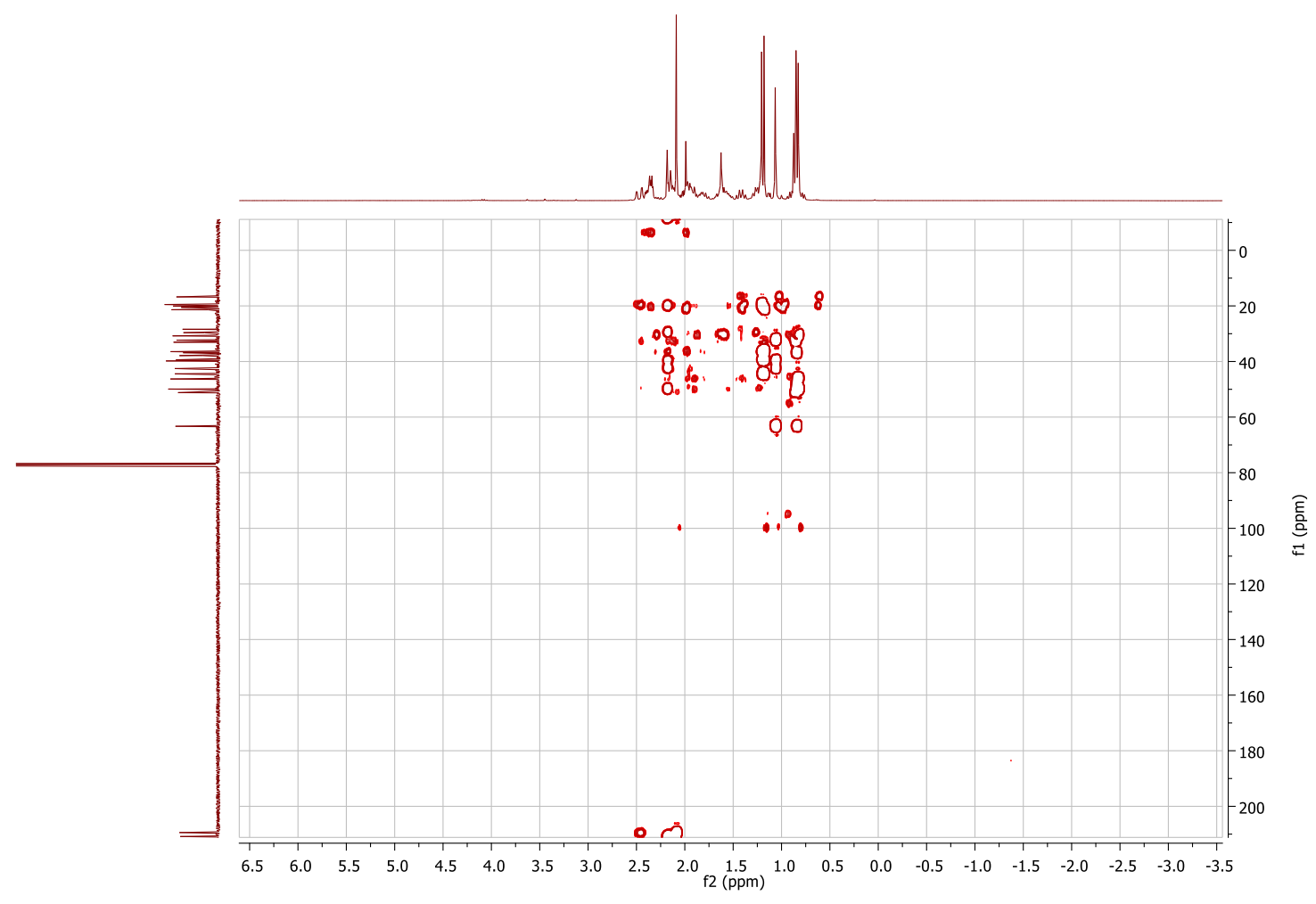

Figure S8. $\mathrm{HMBC}$ spectrum of compound $\mathbf{1}$ in $\mathrm{CDCl}_{3}$

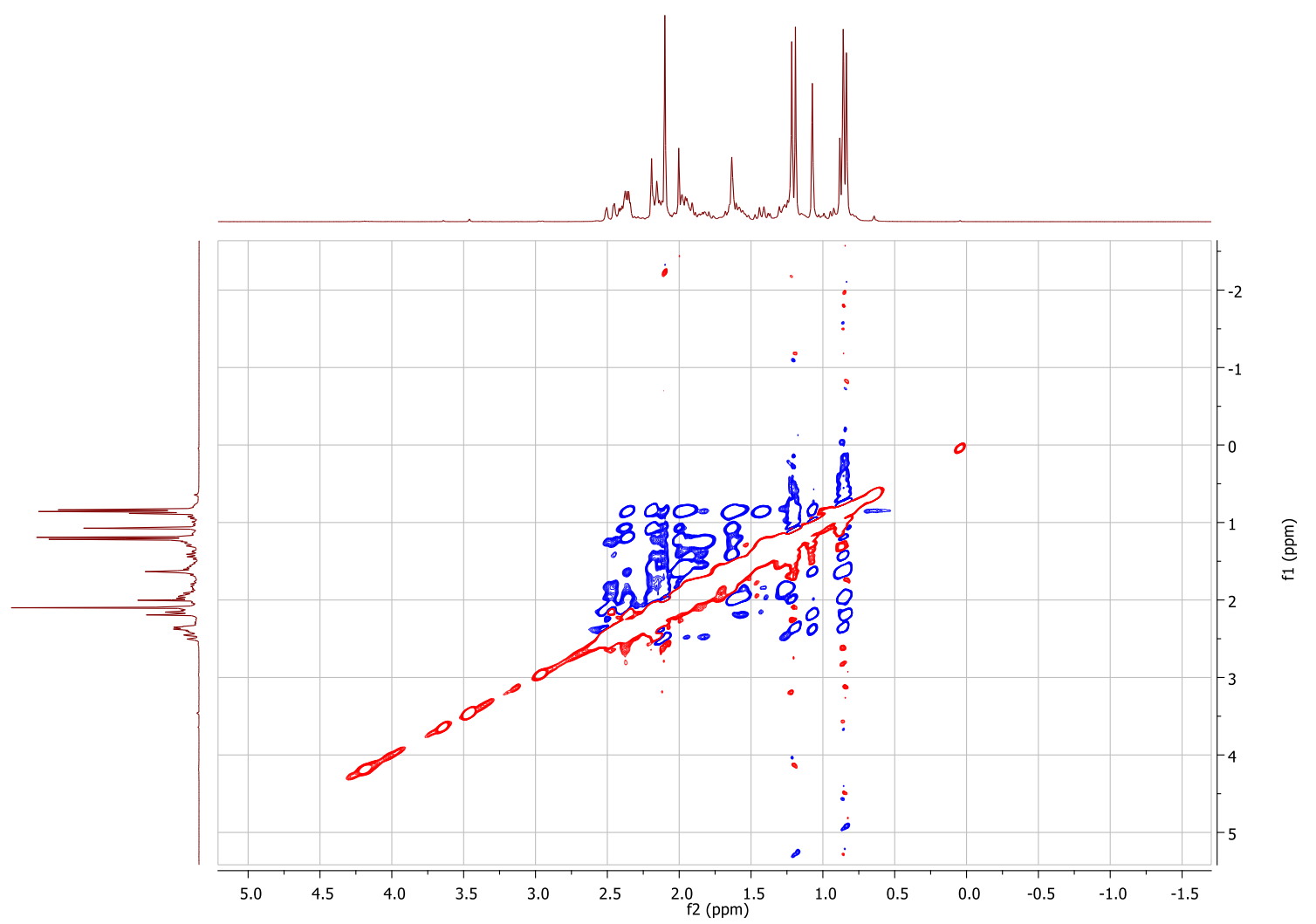

Figure S9. NOESY spectrum of compound $\mathbf{1}$ in $\mathrm{CDCl}_{3}$. 


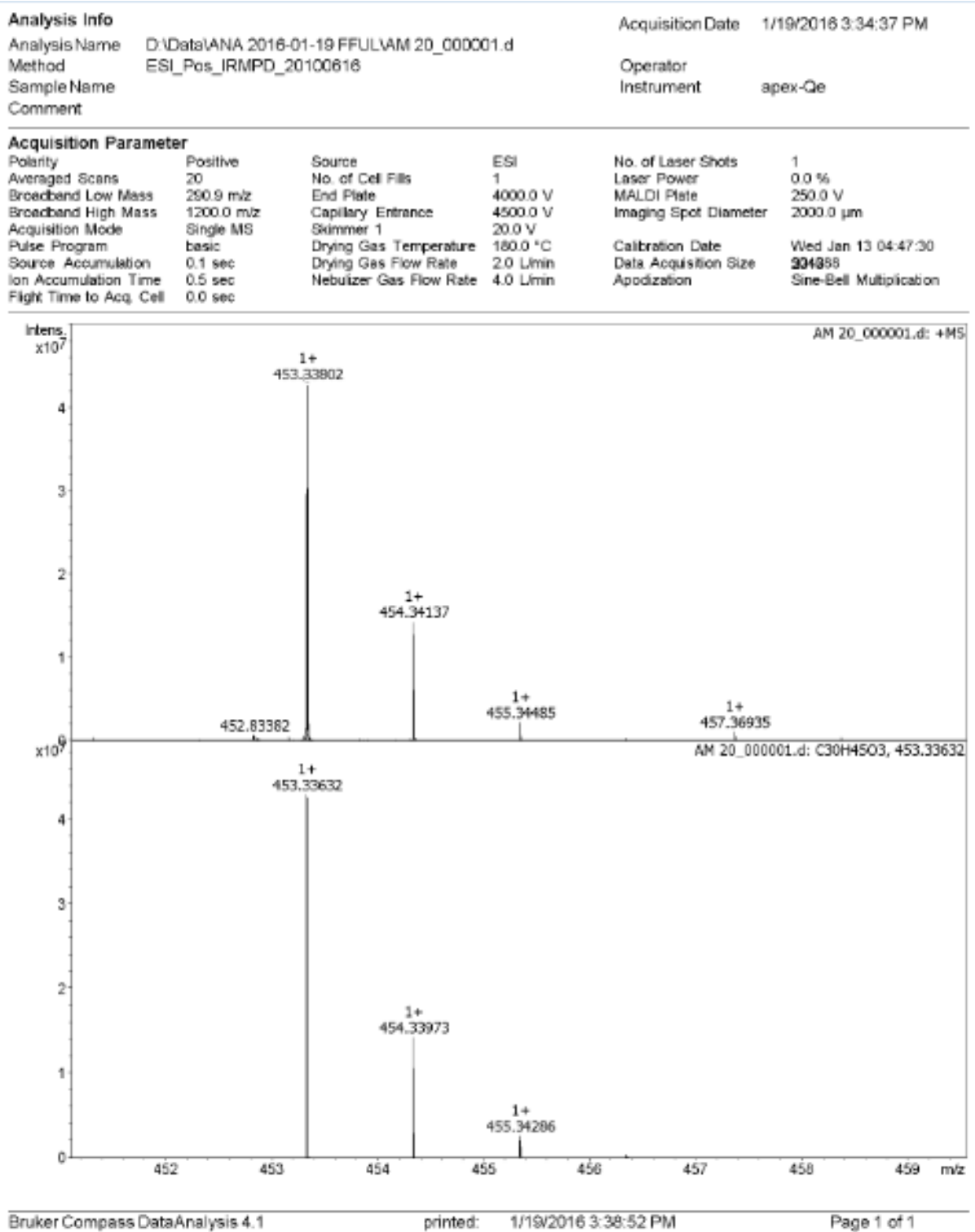

Figure S10. HRMS of compound 2. 
ФSHIMADZU

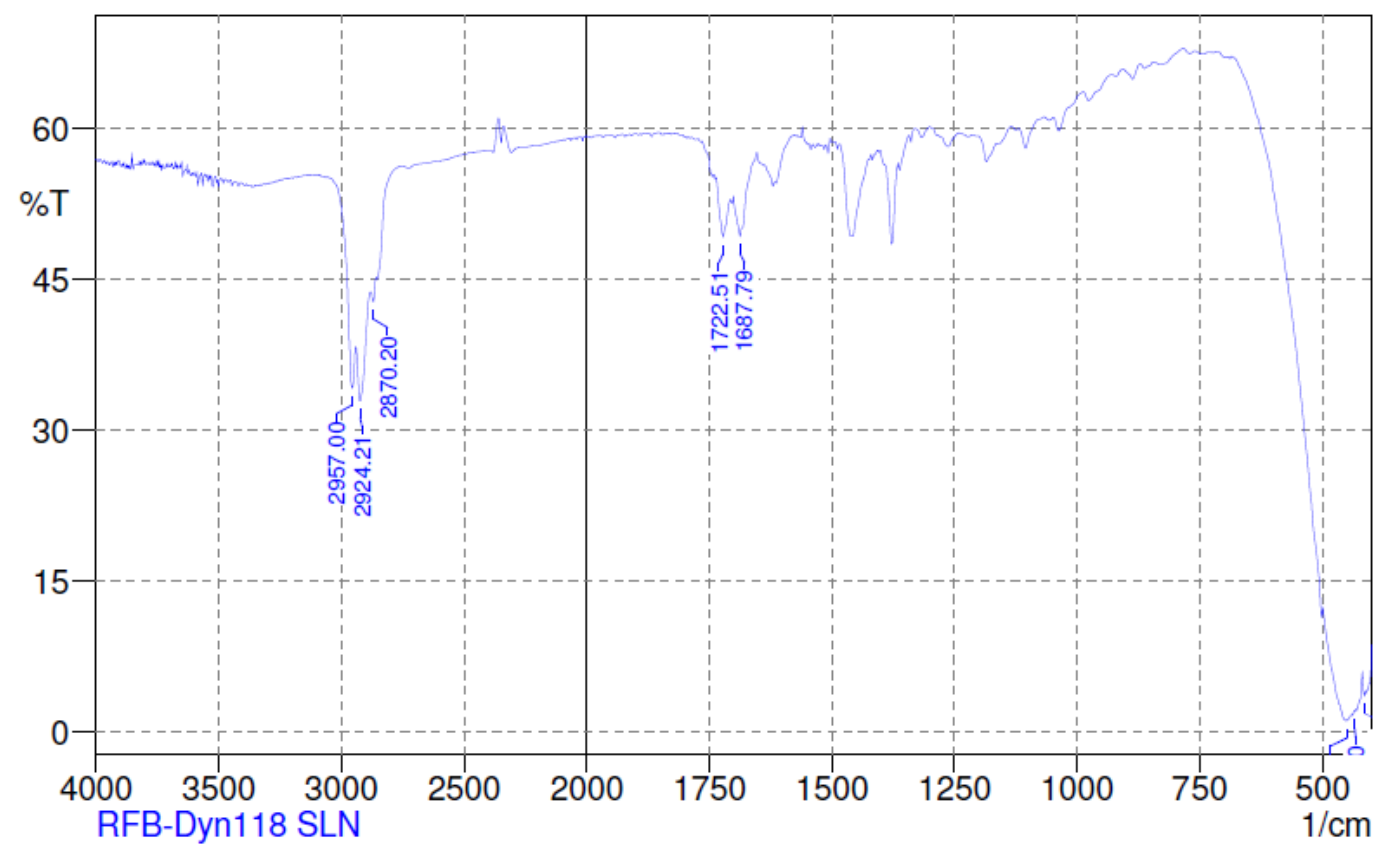

Figure S11. IR of compound 2.

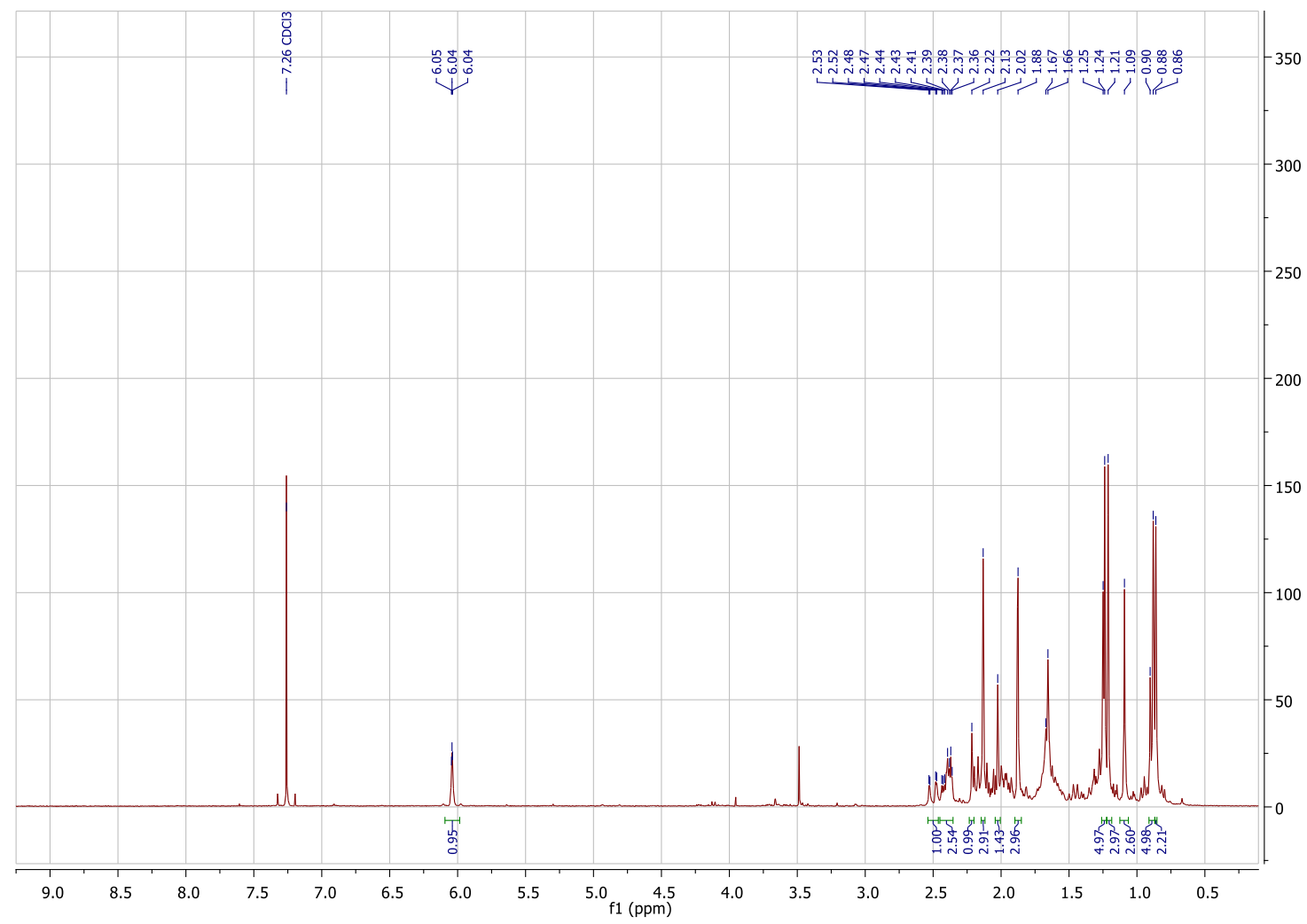

Figure S12. ${ }^{1} \mathrm{H}-\mathrm{NMR}$ spectrum of compound 2 in $\mathrm{CDCl}_{3}(300 \mathrm{MHz})$.. 


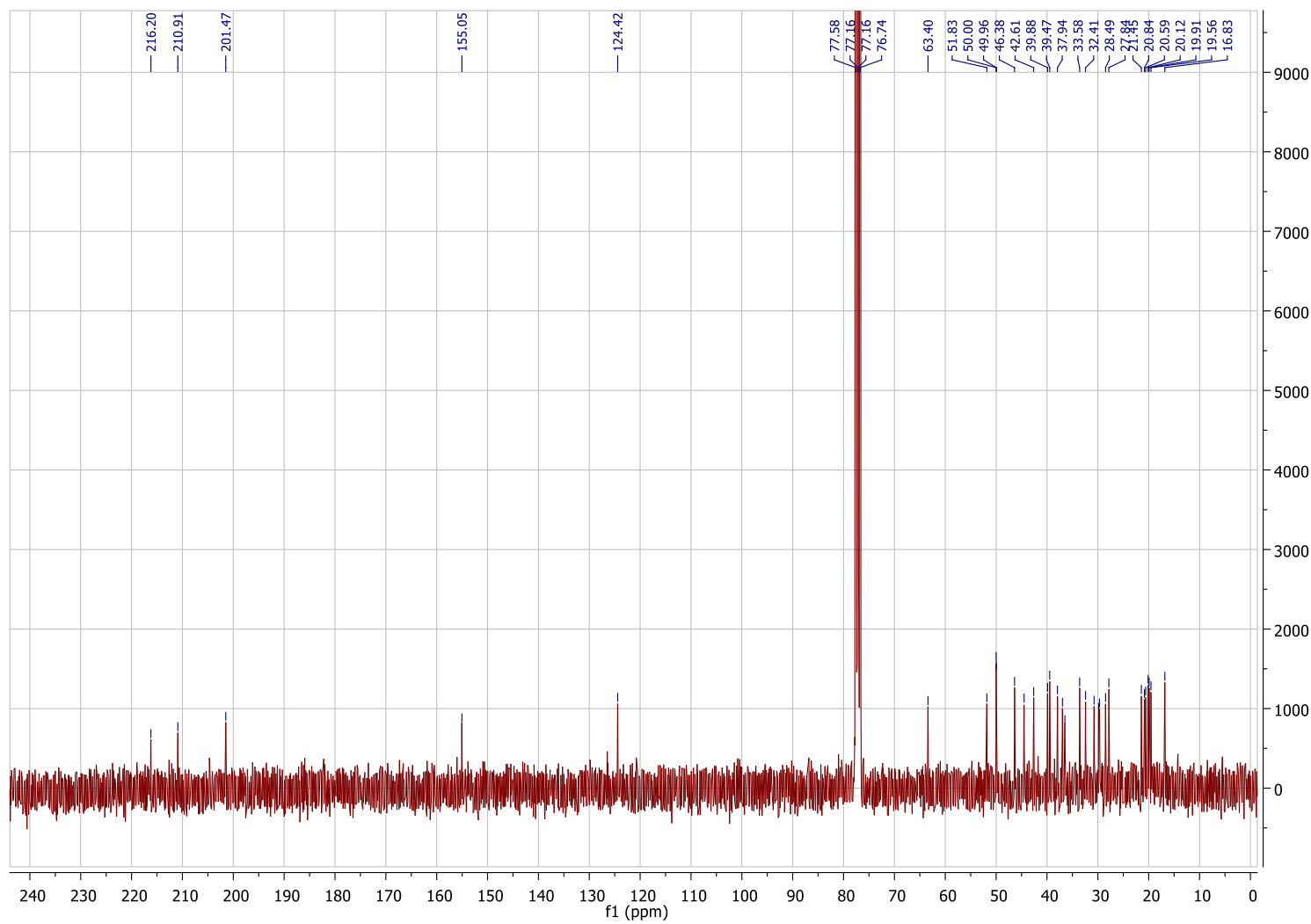

Figure S13. ${ }^{13} \mathrm{C}-\mathrm{NMR}$ spectrum of compound 2 in $\mathrm{CDCl}_{3}(75 \mathrm{MHz})$.

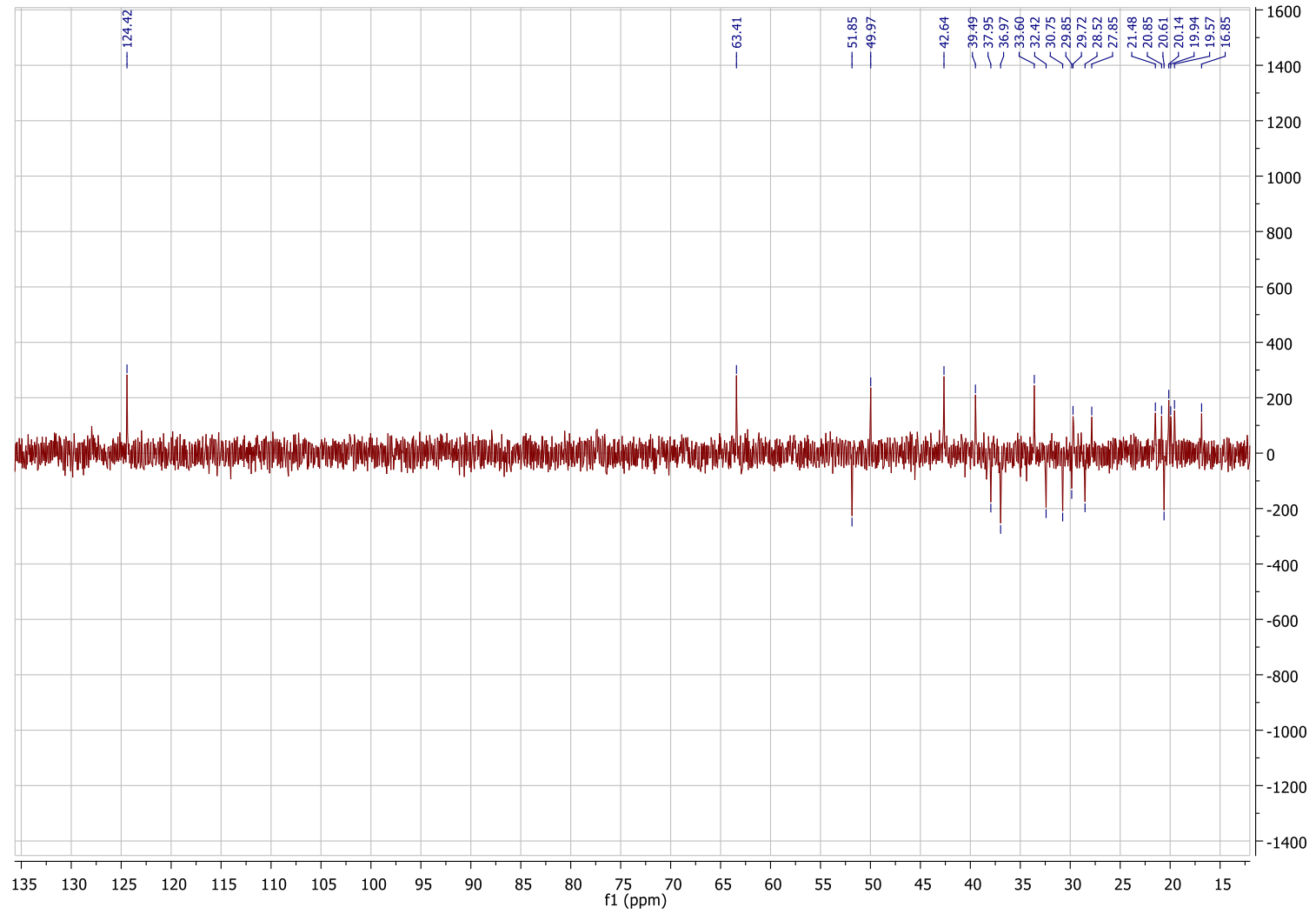

Figure S14. DEPT spectrum of compound 2 in $\mathrm{CDCl}_{3}(75 \mathrm{MHz})$. 


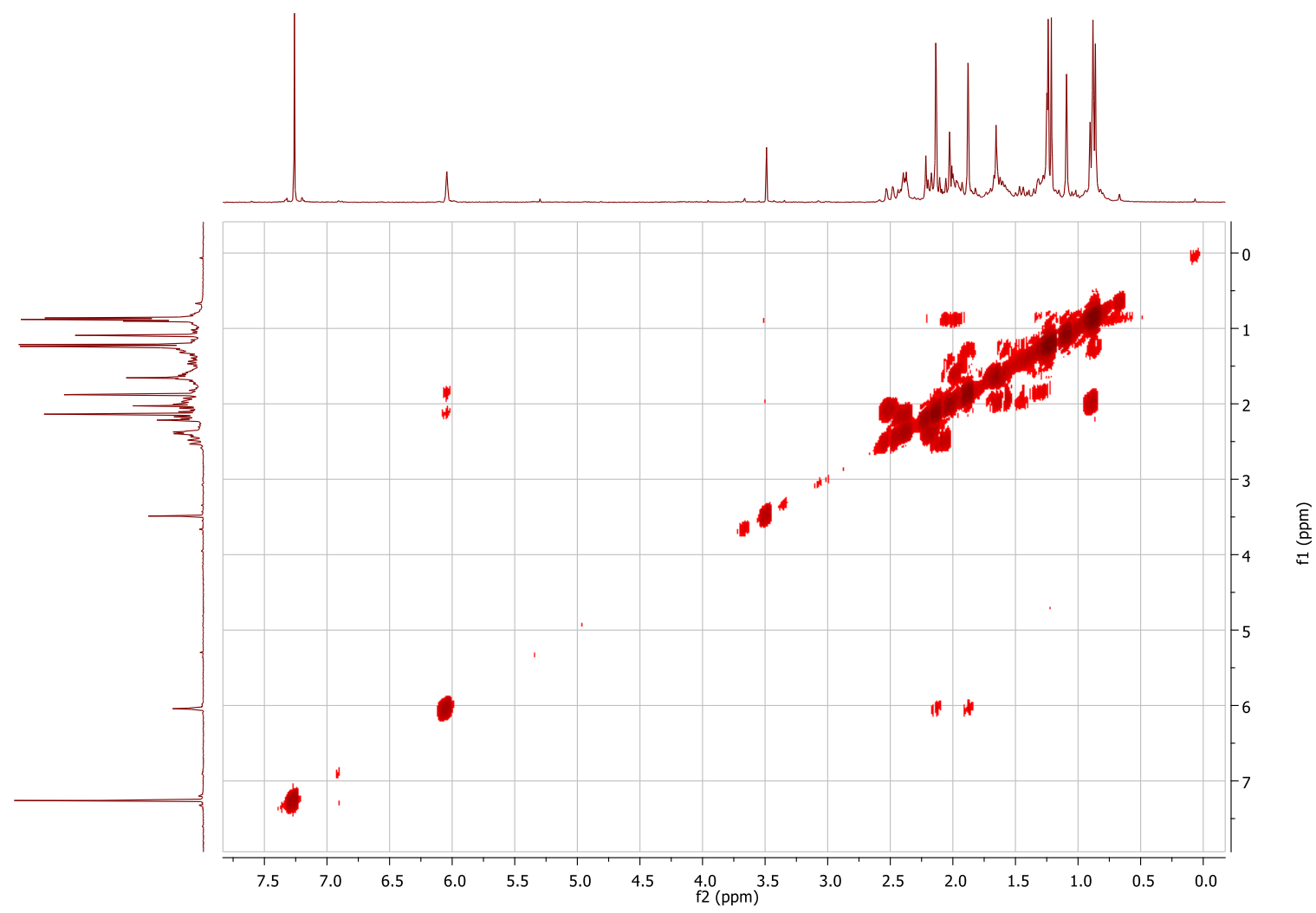

Figure S15. COSY spectrum of compound 2 in $\mathrm{CDCl}_{3}$.

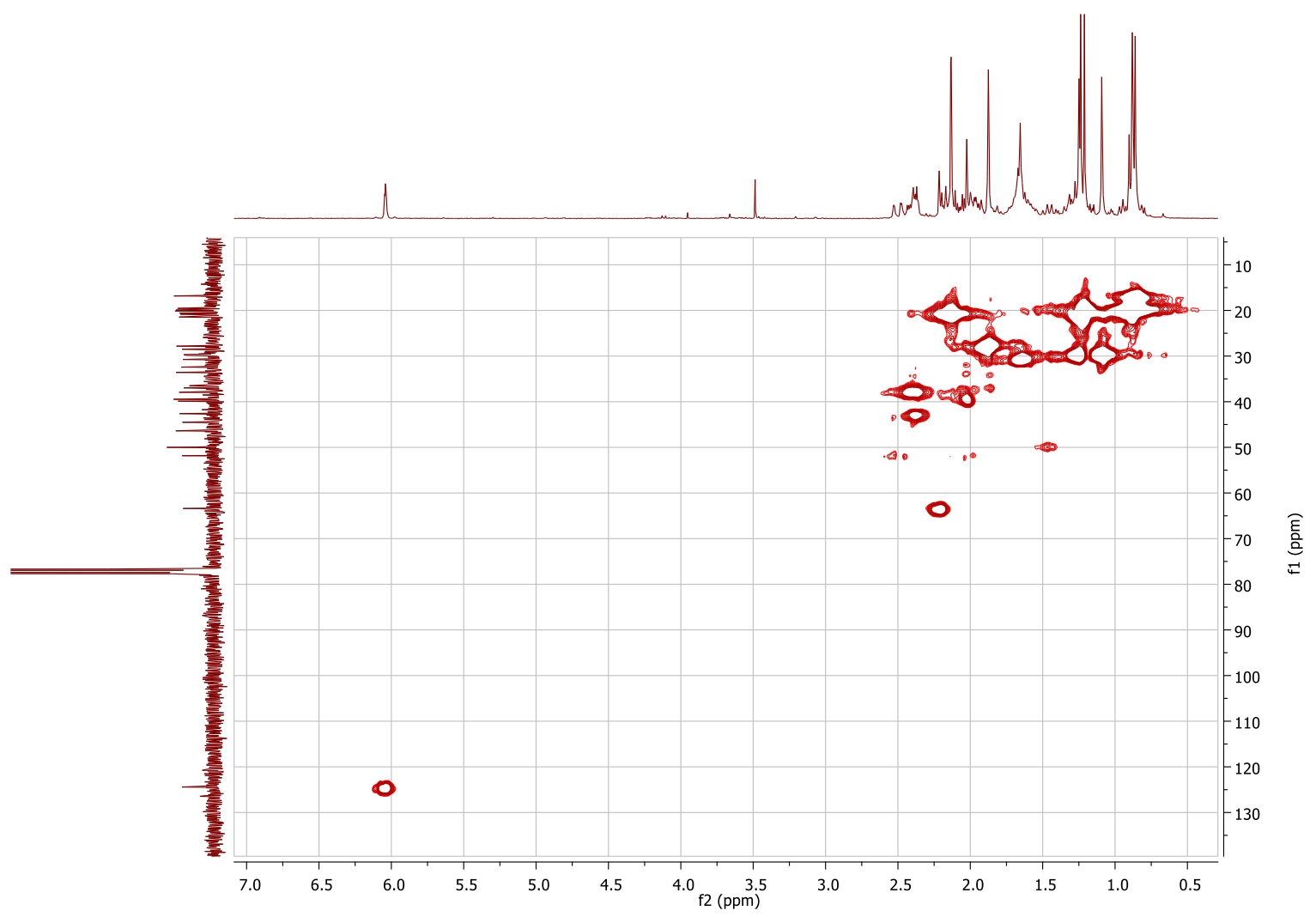

Figure S16. HMQC spectrum of compound 2 in $\mathrm{CDCl}_{3}$. 


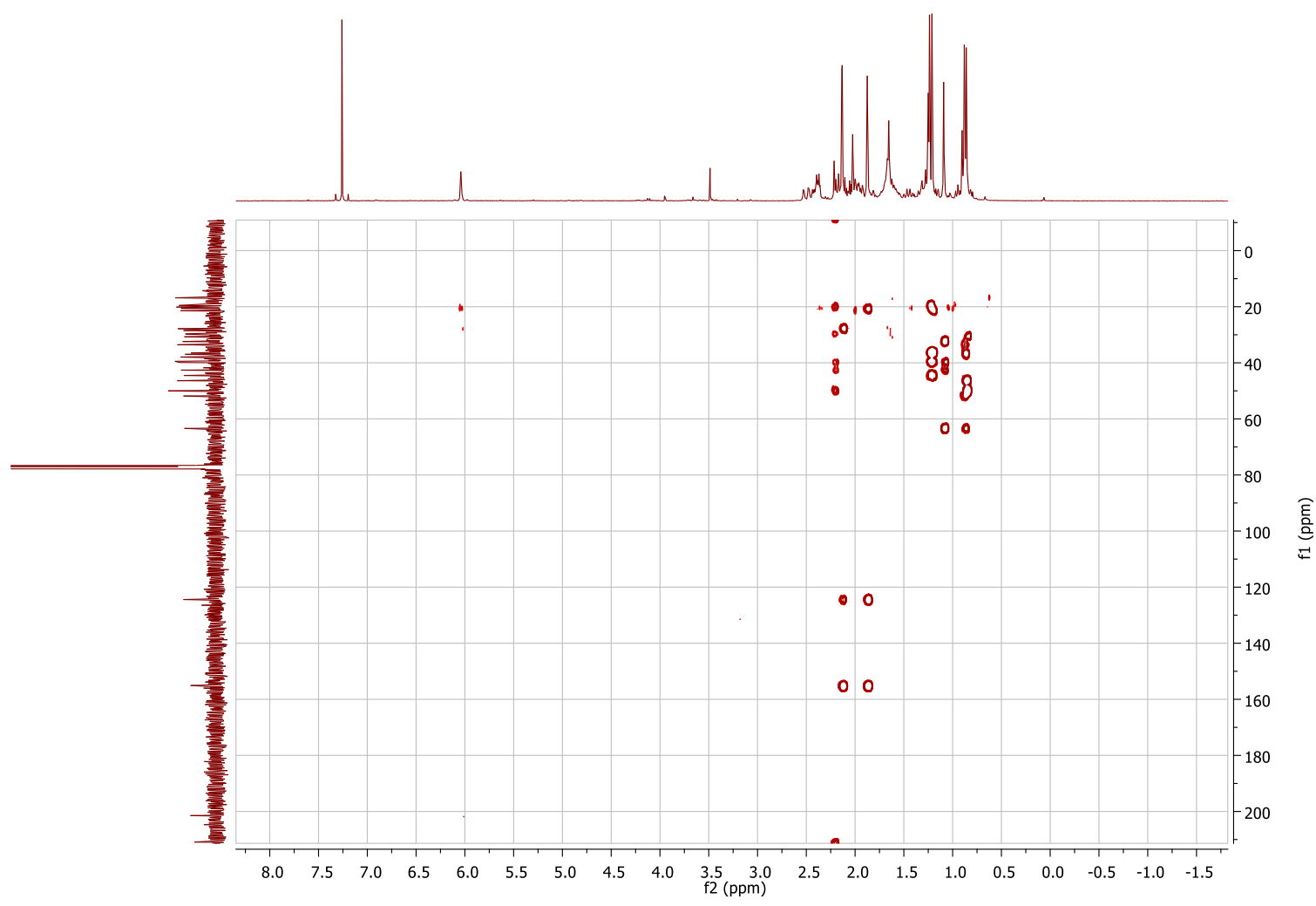

Figure S17. HMBC spectrum of compound 2 in $\mathrm{CDCl}_{3}$.

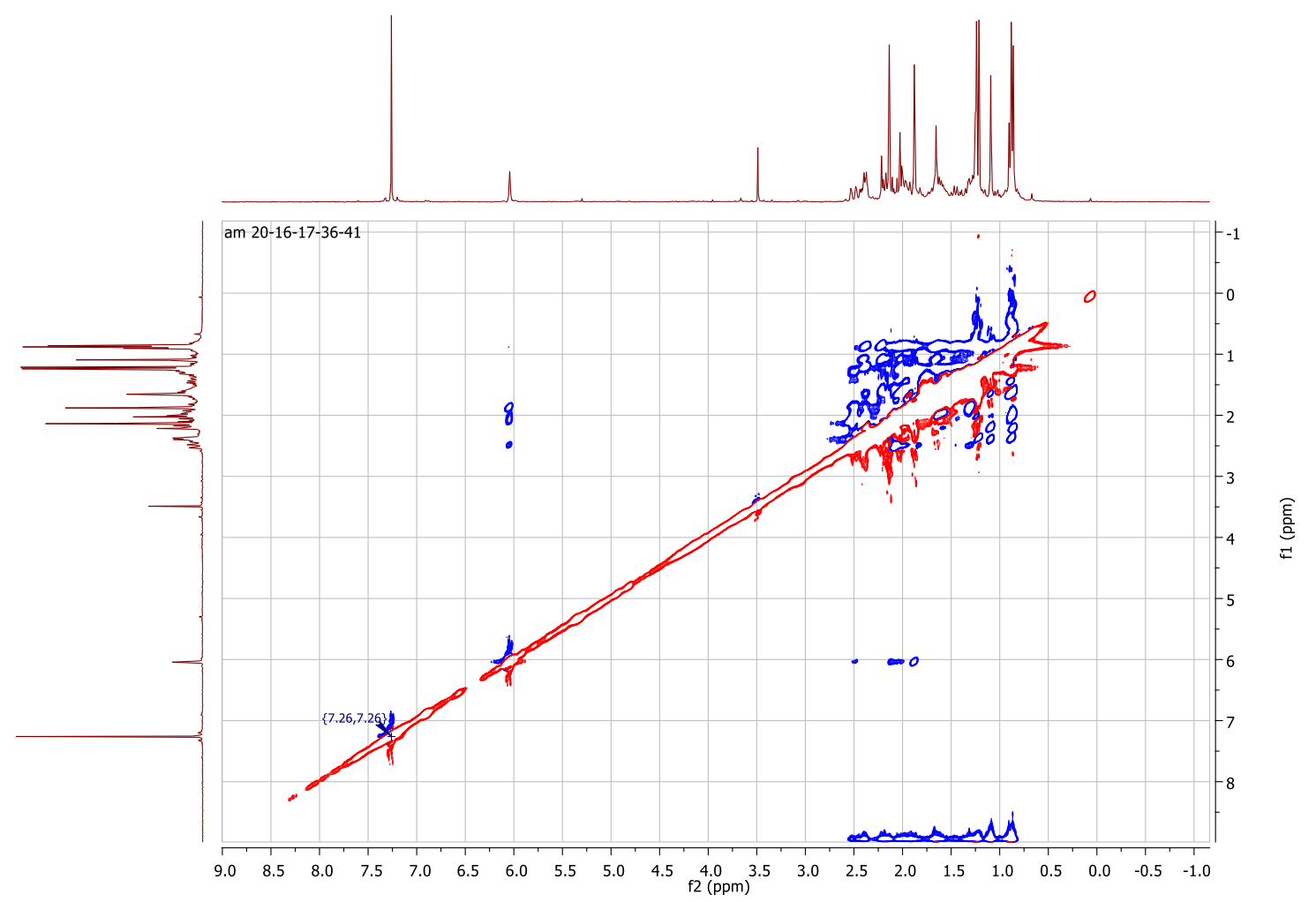

Figure S18. NOESY spectrum of compound 2 in $\mathrm{CDCl}_{3}$. 


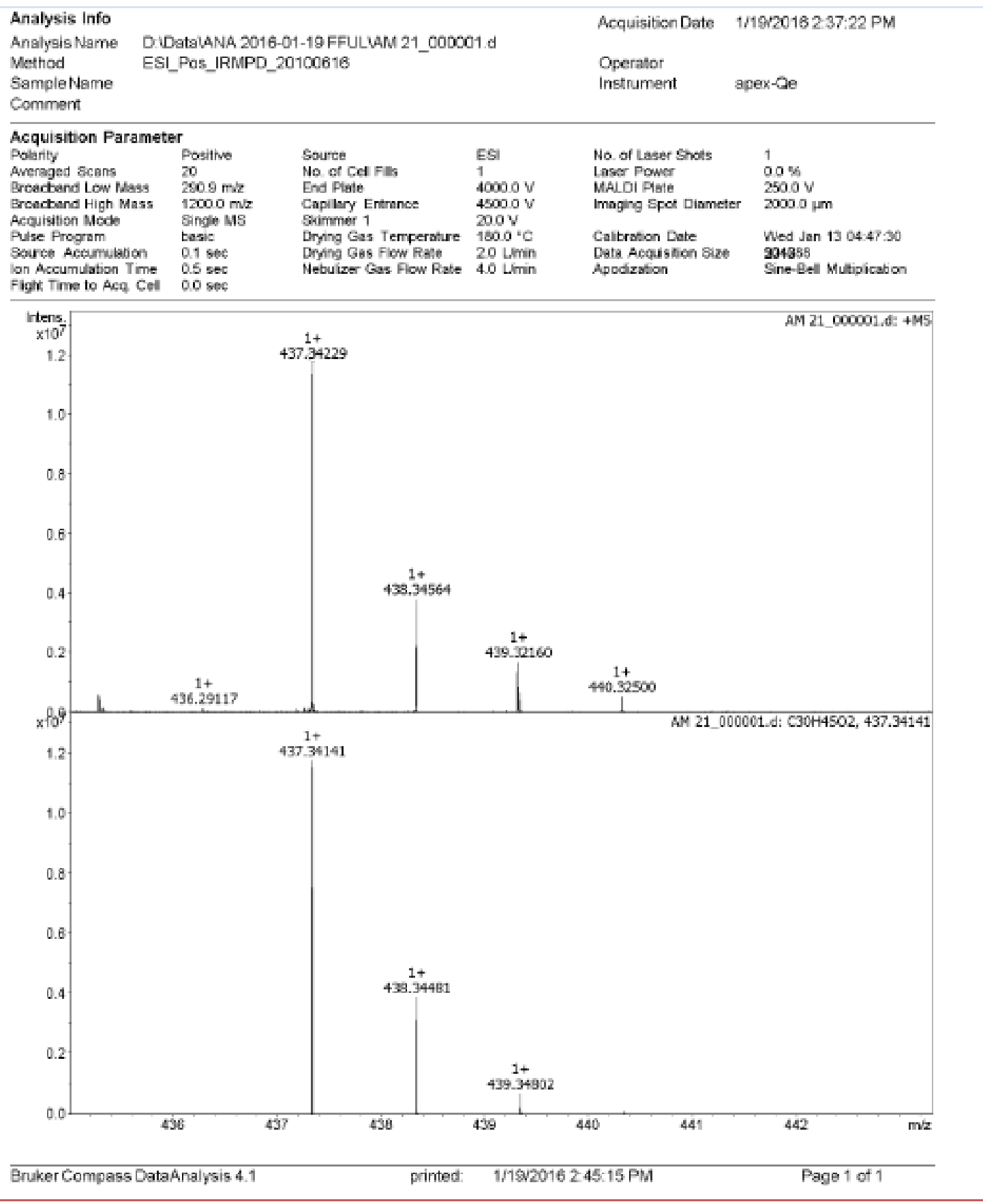

Figure S19. HRMS of compound 3. 
ФSHIMADZU

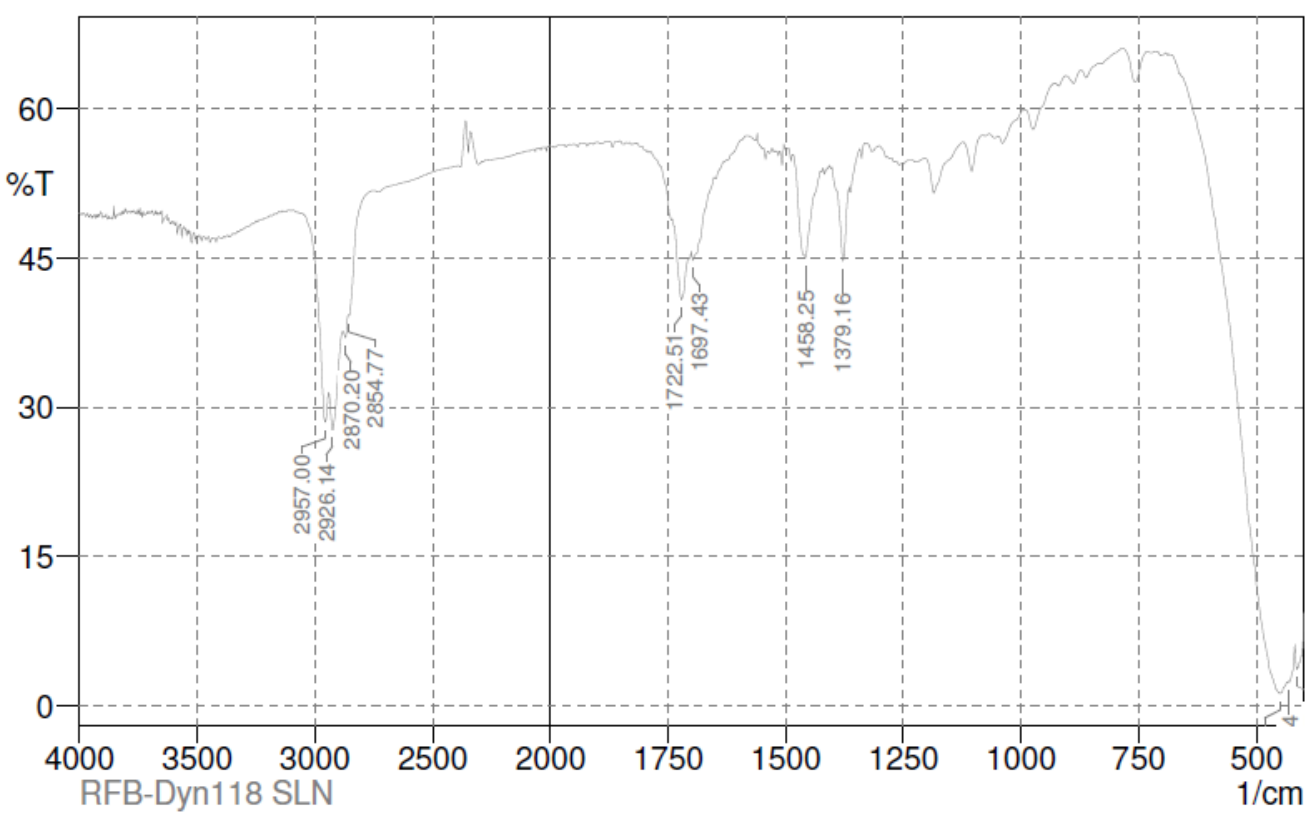

Figure S20. HRMS of compound 3.

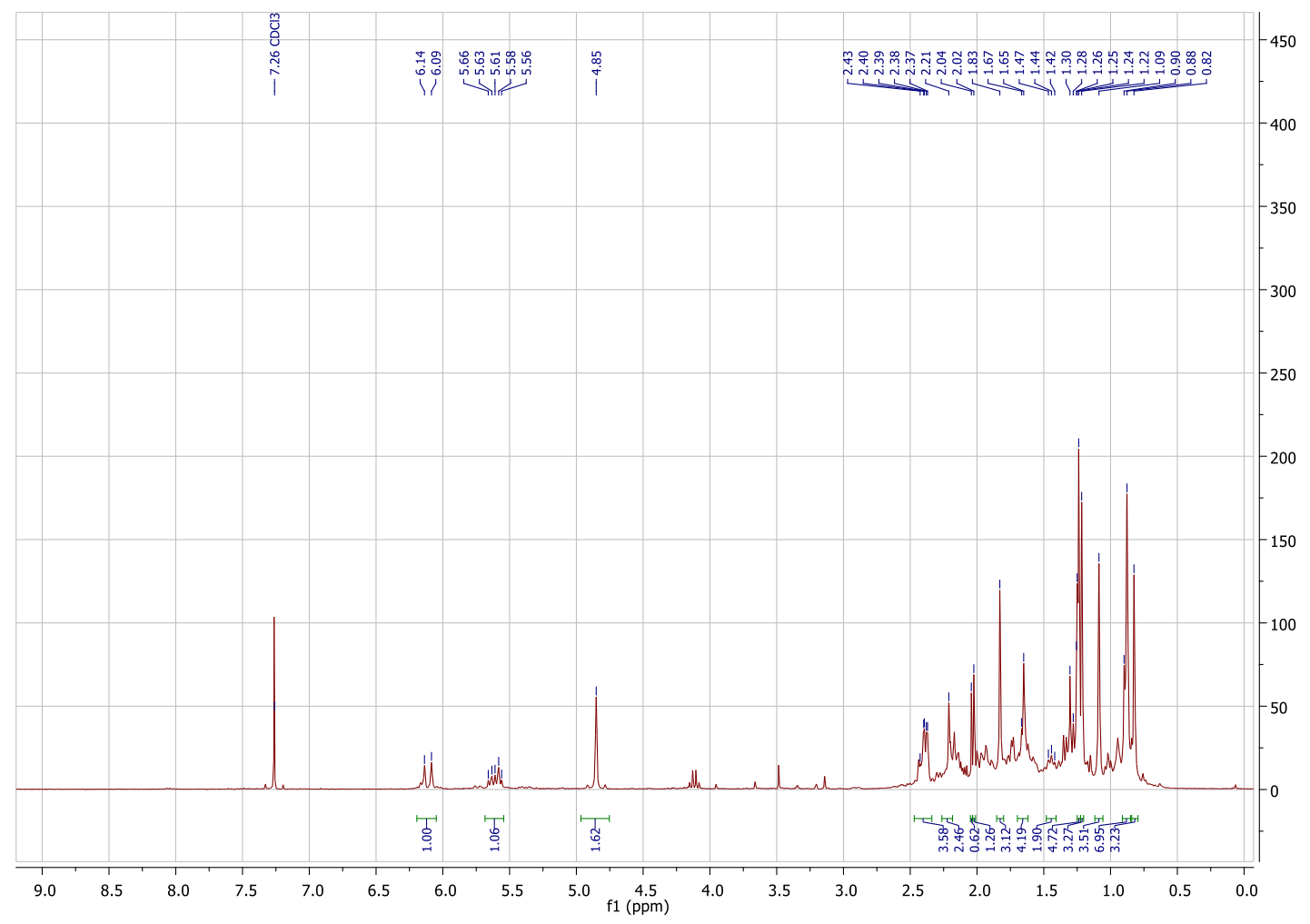

Figure S21 ${ }^{1} \mathrm{H}-\mathrm{NMR}$ spectrum of compound 3 in $\mathrm{CDCl}_{3}(300 \mathrm{MHz})$. 


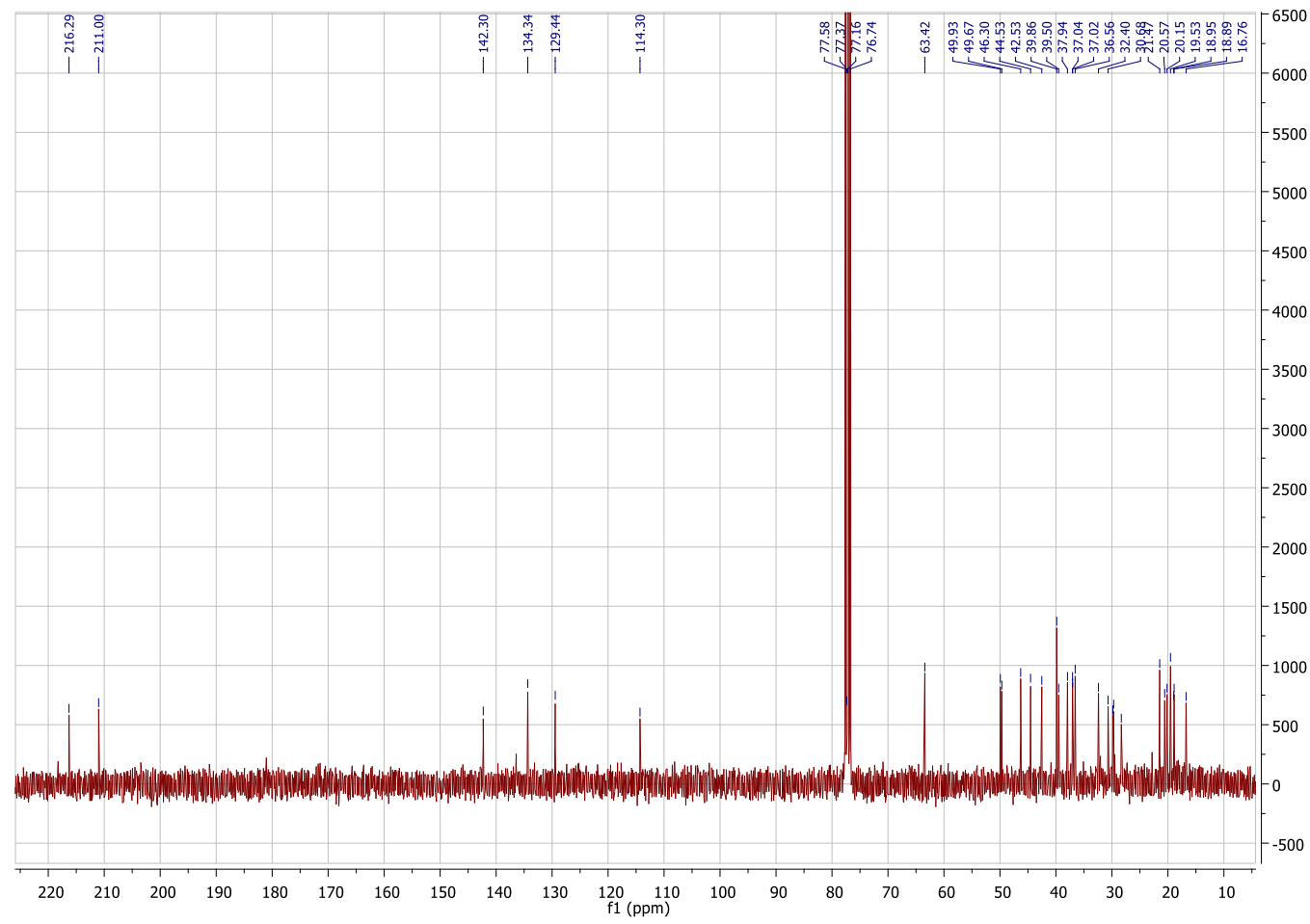

Figure S22. ${ }^{13} \mathrm{C}-\mathrm{NMR}$ spectrum of compound 3 in $\mathrm{CDCl}_{3}(75 \mathrm{MHz})$.

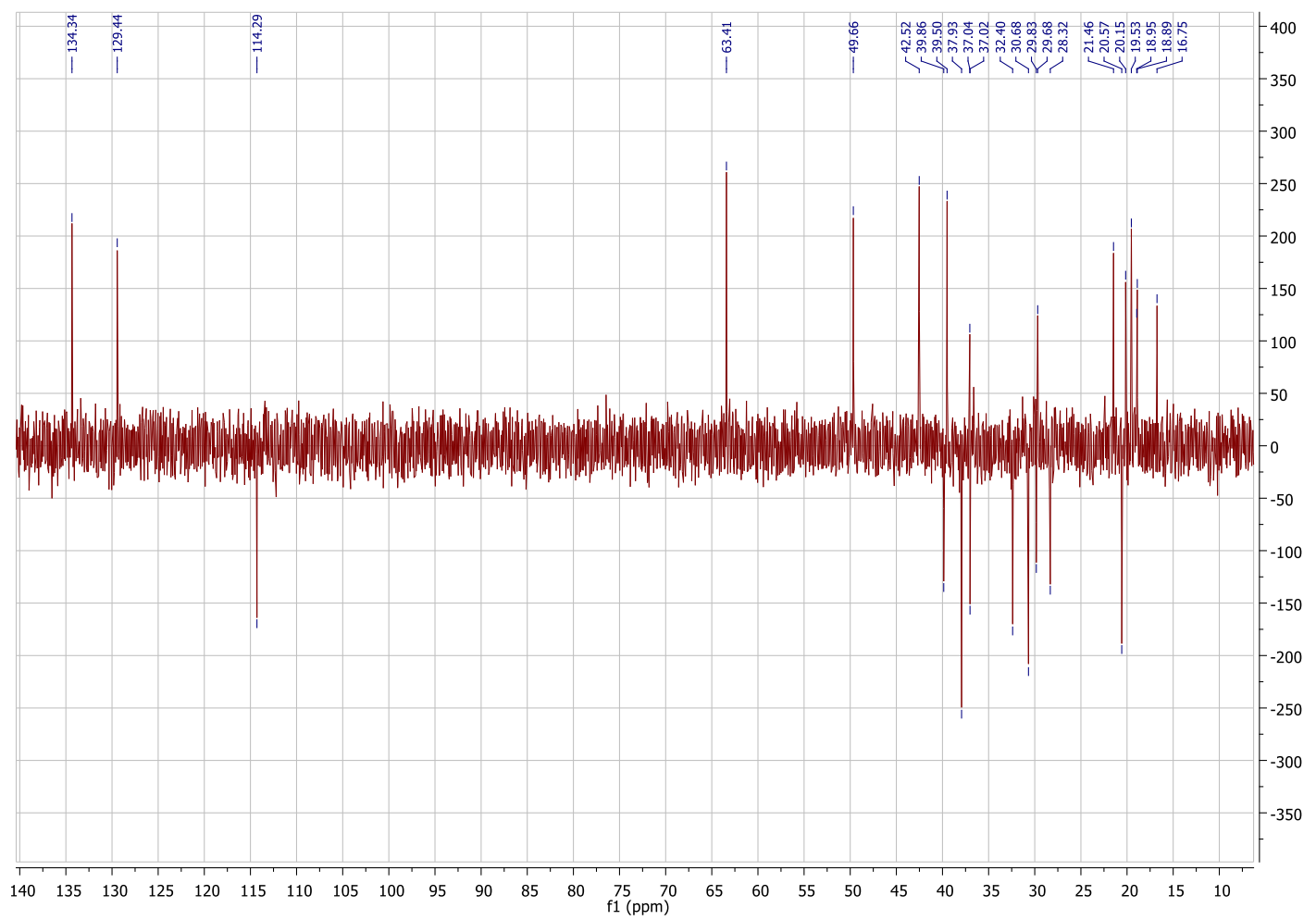

Figure S23. DEPT spectrum of compound 3 in $\mathrm{CDCl}_{3}(75 \mathrm{MHz})$ 


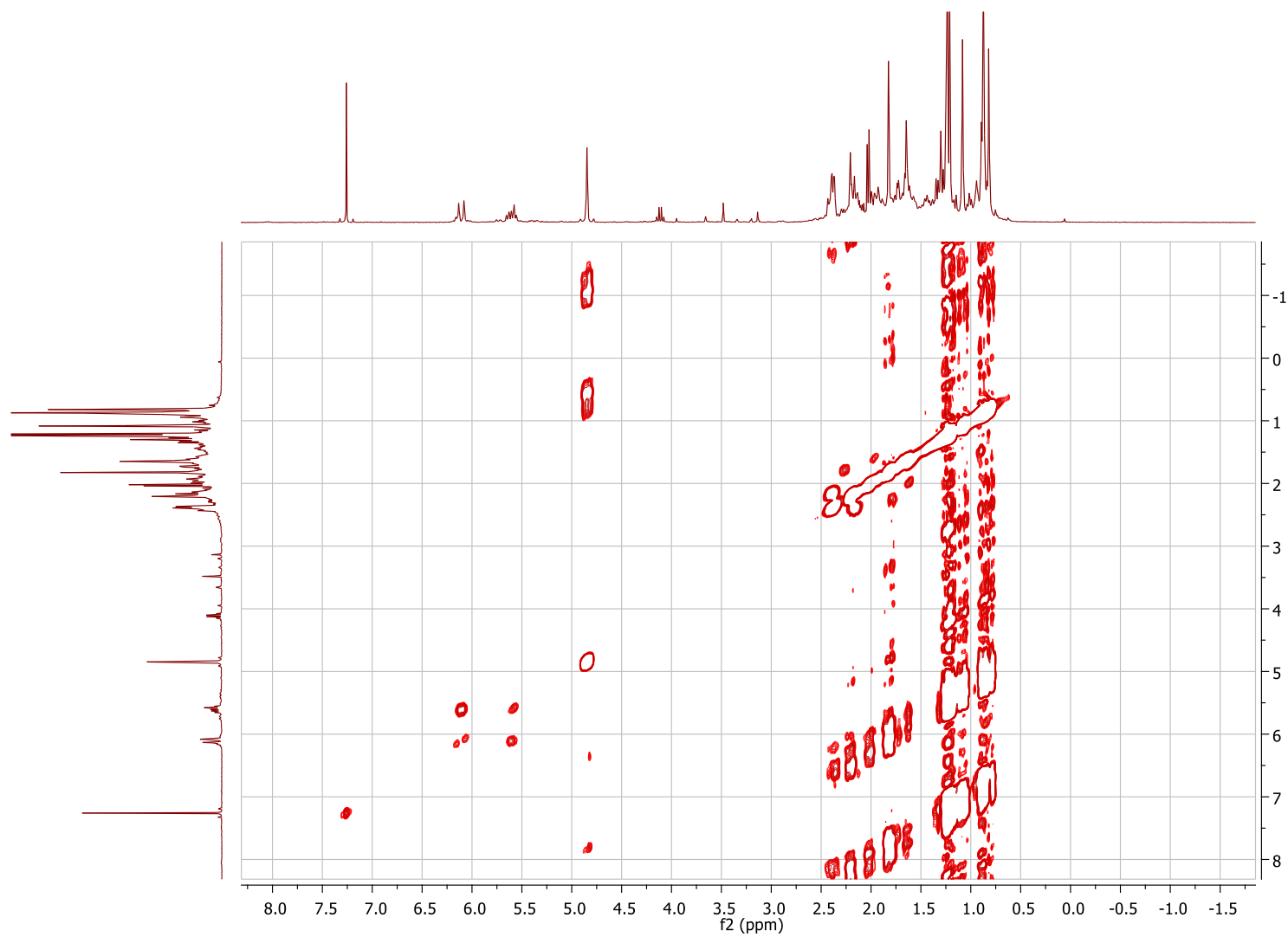

Figure S24. COSY spectrum of compound 3 in $\mathrm{CDCl}_{3}$.

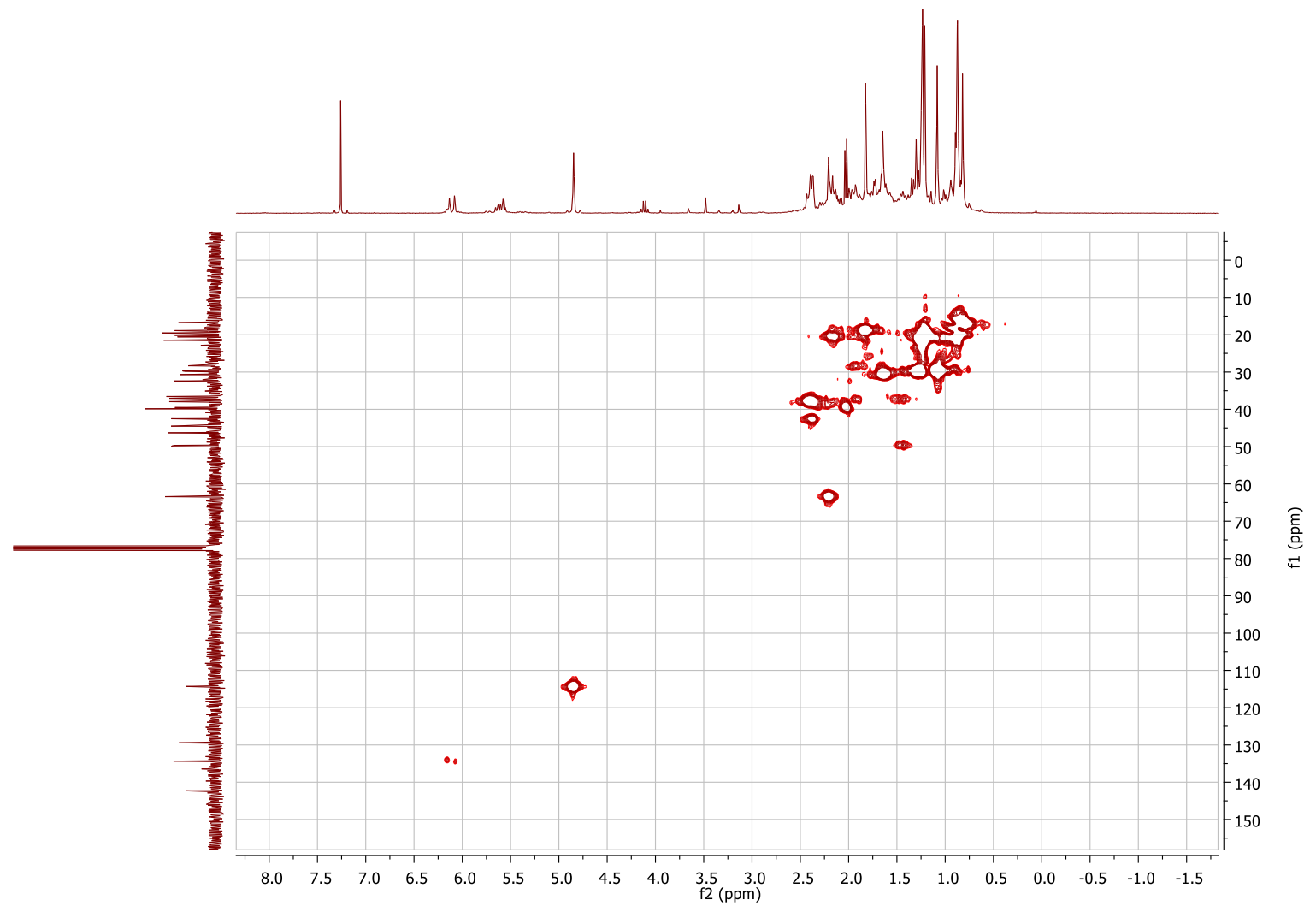

Figure S25. HMQC spectrum of compound $\mathbf{3}$ in $\mathrm{CDCl}_{3}$. 


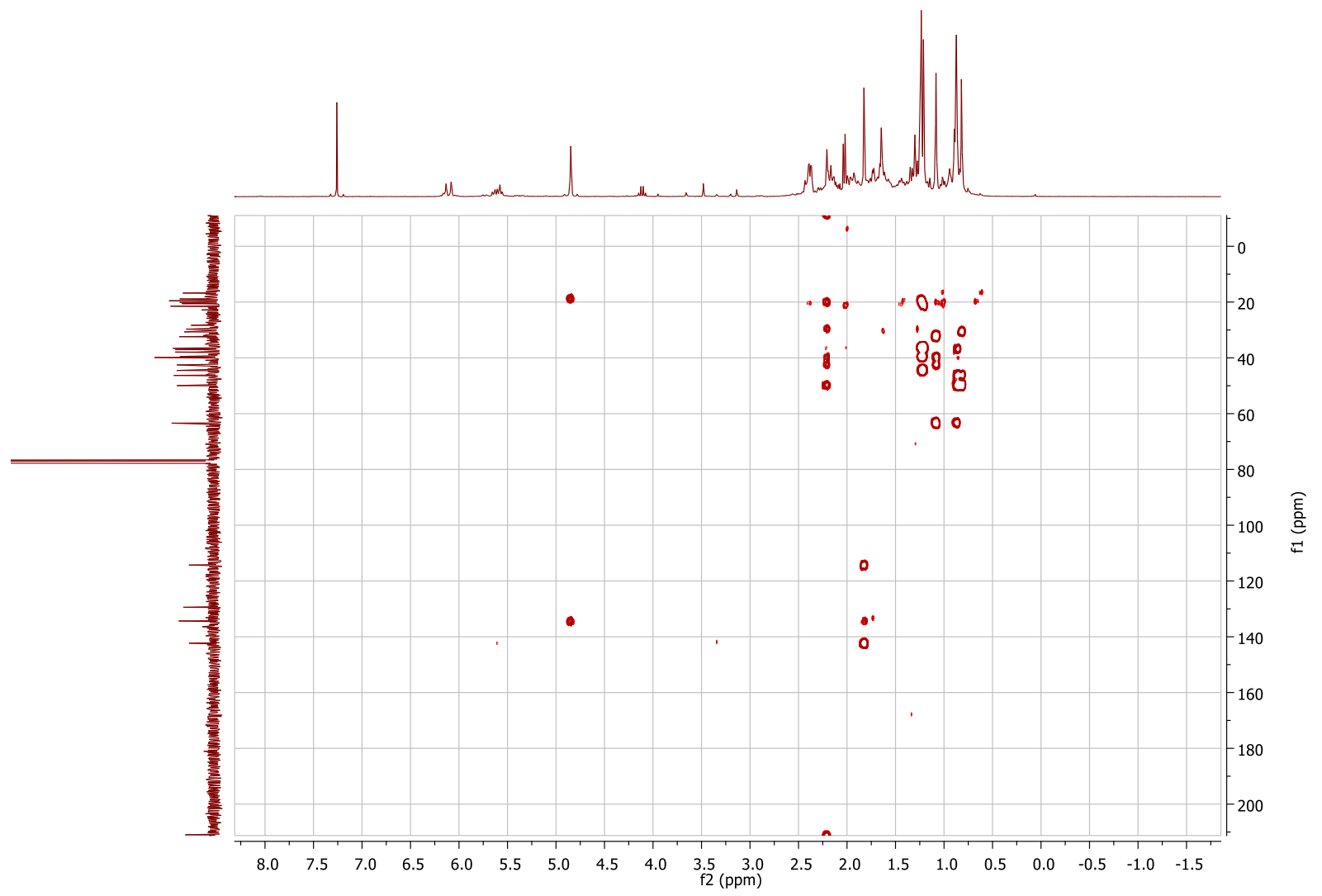

Figure S26. $\mathrm{HMBC}$ spectrum of compound $\mathbf{3}$ in $\mathrm{CDCl}_{3}$ 